\title{
Application de la diffusion inélastique aux systèmes désordonnés
}

\author{
M. Bée
}

Institut Laue-Langevin, 6 rue Jules Horowitz, BP. 159, 38042 Grenoble cedex 9, France

\begin{abstract}
Résumé. La très grande section efficace incohérente de l'hydrogène, comparée à celle des autres éléments, fait de la diffusion des neutrons une technique privilégiée pour l'analyse des mouvements individuels des protons dans les composés hydrogénés. Nous analysons les origines du déplacement d'un atome diffuseur (vibrations intramoléculaires, modes de réseau, réorientations de groupements chimiques ou rotation d'ensemble des molécules, déplacement de translation). Sous réserve de pouvoir négliger les corrélations entre ces différentes composantes leurs contributions à l'intensité diffusée peuvent être séparées et il est possible de calculer pour chacune la fonction de diffusion dynamique correspondante, la fonction de diffusion dynamique globale résultant de la convolution de ces différentes expressions. Nous montrons comment il est possible d'accéder à la fonction de diffusion quasiélastique dont l'élargissement est directement reliée aux temps caractéristiques des mouvements sur la gamme de temps $10^{-10} \cdot 10^{-12} \mathrm{~s}$. La part de l'intensité diffusée purement élastique renseigne quant à elle sur la géométrie de ces mouvements. Nous présentons rapidement les modèles les plus couramment utilisés. Des exemples viennent en illustration de ces considérations théoriques, et permettent d'en préciser les limites de validité.
\end{abstract}

\section{PROPRIETES DU NEUTRON}

Les neutrons issus du cœur du réacteur nucléaire sont thermalisés en passant au travers d'un modérateur à la température $T$. Dès lors la distribution de leurs vitesses suit une loi de Maxwell autour d'une valeur moyenne telle que :

$$
E=\frac{1}{2} m^{2}=\frac{3}{2} k_{B} T
$$

$k_{B}$ étant la constante de Boltzmann et $m$ la masse du neutron $\left(m=1,66010^{-27} \mathrm{~kg}\right)$. Les neutrons peuvent aussi être considérés comme des ondes planes, de vecteur d'onde

$$
\boldsymbol{k}=\frac{m}{\hbar}
$$

et de longueur d'onde

$$
\lambda=\frac{2 \pi}{|\boldsymbol{k}|}=\frac{h}{m}
$$

de sorte que l'énergie cinétique s'exprime comme

$$
E=\frac{\hbar^{2} k^{2}}{2 m}=\frac{h^{2}}{2 m \lambda^{2}}
$$

Les neutrons thermiques, correspondant à $T=300 \mathrm{~K}$, ont une énergie d'environ $25 \mathrm{meV}$ (milliélectronvolts) et une longueur d'onde de $1,8 \AA$ ( $=2200 \mathrm{~m} / \mathrm{s})$. Les « sources froides » ou « sources chaudes » thermalisent les neutrons à $25 \mathrm{~K}$ ou $2000 \mathrm{~K}$ et permettent de déplacer le maximum de la distribution respectivement vers les grandes longueurs d'onde $(3,5 \AA)$ ou vers les courtes longueurs d'onde $(0,5 \AA)$.

De ces valeurs on retiendra surtout que l'énergie des neutrons thermiques apparaît du même ordre de grandeur que les énergies intermoléculaires dans les phases condensées êt que leur longueur d'onde est 
comparable aux dimensions moléculaires usuelles et aux distances interréticulaires dans les solides. Ces deux propriétés font du neutron un outil très puissant pour l'étude microscopique des propriétés tant dynamiques que géométriques de la matière. L'énergie des neutrons froids ou thermiques est certes trop faible pour provoquer des excitations internes du noyau ou des couches électroniques. Néanmoins le neutron est sensible aux déplacements atomiques des noyaux qui correspondent à des énergies beaucoup plus faibles et qui sont à l'origine de la diffusion inélastique.

\section{LES FONCTIONS DE DIFFUSION NEUTRONIQUES}

Le schéma général d'une expérience de diffusion de neutrons est représenté sur la figure 1.

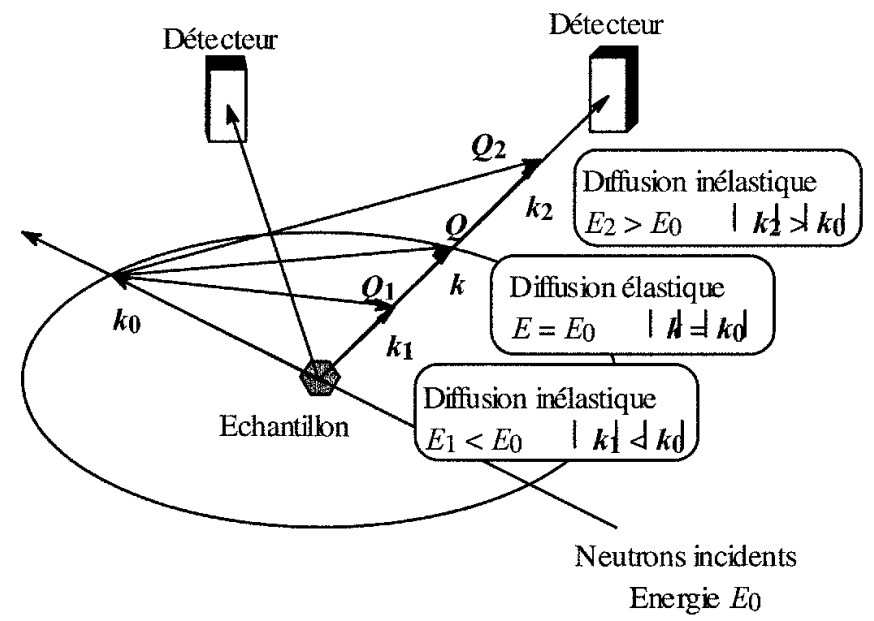

Figure 1: Schéma général d'une expérience de diffusion de neutrons. Pour une même valeur de l'angle de diffusion, le vecteur de diffusion, $Q$, varie en fonction de l'énergie échangée par le neutron avec l'atome diffuseur.

Les deux principales grandeurs mesurées sont:

i) le vecteur de diffusion, $\boldsymbol{Q}$, différence entre le vecteur d'onde final du neutron, $\boldsymbol{k}$, et son vecteur d'onde initial, $\boldsymbol{k}_{\theta}$

$$
Q=k-k_{0}
$$

ii) la différence, $\hbar \omega$, entre les valeurs finales, $E$, et initiale, $E_{0}$, de l'énergie du neutron,

$$
\hbar \omega=E-E_{0}=\frac{\hbar^{2}}{2 m}\left(k^{2}-k_{0}^{2}\right)
$$

La formule de base de la diffusion nucléaire, donnant la probabilité qu'un neutron, arrivant sur un ensemble de $N$ diffuseurs avec l'énergie incidente $E_{0}$ et un vecteur d'onde $\boldsymbol{k}_{\mathbf{0}}$ soit diffusé avec le vecteur d'onde $k$ dans l'élément d'angle solide $d \Omega$ autour de la direction de $k$ et avec une énergie comprise entre $E$ et $E+\hbar d \Omega$ est

$$
\frac{d^{2} \sigma}{d \Omega d \omega}=\frac{k}{k_{0}} \frac{1}{N} \sum_{\alpha} \sum_{\beta} \frac{1}{2 \pi} \int_{-\infty}^{\infty}\left\langle b_{\alpha} b_{\beta} \cdot e^{-i Q \cdot r_{\alpha}(0)} e^{i Q \cdot r_{\beta}(t)}\right\rangle e^{-i \omega t} d t
$$


Chacune des sommes sur les indices $\alpha$ et $\beta$ porte sur tous les atomes de l'échantillon. Les termes $b_{\alpha}$ et $b_{\beta}$ sont les longueurs de diffusion, qui caractérisent l'interaction neutron - noyau de nature à la fois nucléaire et magnétique. La longueur de diffusion change non seulement en passant d'une espèce chimique à l'autre, mais aussi pour les différents isotopes de la même espèce, car l'interaction neutron noyau dépend non seulement de la nature de ce dernier mais aussi de l'état de spin total du système. Lors d'une expérience, l'échantillon est composé de différentes espèces atomiques, $i$, chacune d'elle pouvant être un mélange de plusieurs isotopes possédant leur propre spin nucléaire. Les crochets désignent une moyenne thermique qui porte à la fois sur les positions $\boldsymbol{r}_{\alpha}(0)$ et $\boldsymbol{r}_{\beta}(t)$ des noyaux de l'échantillon et sur tous les états de spin. En général, il n'existe pas de couplage entre la longueur de diffusion d'un noyau et sa position de sorte que les moyennes sur les états de spin et sur les coordonnées peuvent être prises séparément

$$
\frac{d^{2} \sigma}{d \Omega d \omega}=\frac{k}{k_{0}} \frac{1}{N} \sum_{\alpha} \sum_{\beta} \overline{b_{\alpha} b_{\beta}} \frac{1}{2 \pi} \int_{-\infty}^{\infty}\left\langle e^{-i Q \cdot r_{\alpha}(0)} e^{i Q \cdot r_{\beta}(t)}\right\rangle e^{-i \omega t} d t
$$

Cette expression dans laquelle la moyenne thermique ne porte plus que sur les coordonnées, est valable pour un système composé de plusieurs types de diffuseurs. Les sommes sur les indices $\alpha$ et $\beta$ des atomes portent maintenant sur les différentes espèces chimiques et sur les isotopes de chaque espèce. En réalité, dans ce cours nous considérerons essentiellement la diffusion incohérente par des composés organiques hydrogénés. Compte tenu des propriétés particulières de la longueur de diffusion de l'hydrogène, on est le plus souvent amené dans ce type d'échantillons à ne considérer que la présence de l'hydrogène seul. C'est pourquoi nous limiterons notre introduction des grandeurs fondamentales de la diffusion des neutrons dans l'hypothèse d'un système à un seul composant. Pour plus d'information concernant le cas des systèmes à plusieurs composants, nous renvoyons à la littérature $[1,6]$. Signalons que

$$
\sigma=\int d \omega \int d \Omega \frac{\partial^{2} \sigma}{\partial \omega \partial \Omega}
$$

L'équation (8) peut s'écrire sous la forme

$$
\begin{aligned}
\frac{d^{2} \sigma}{d \Omega d \omega} & =\frac{k}{k_{0}} \bar{b}^{2} \frac{1}{N} \sum_{\alpha} \sum_{\beta} \frac{1}{2 \pi} \int_{-\infty}^{\infty}\left\langle\cdot e^{-i Q \cdot r_{\alpha}(0)} e^{i Q \cdot n_{\beta}(t)}\right\rangle e^{-i \omega t} d t \\
& +\frac{k}{k_{0}}\left(\overline{b^{2}}-\bar{b}^{2}\right) \frac{1}{N} \sum_{\alpha} \frac{1}{2 \pi} \int_{-\infty}^{\infty}\left\langle\cdot e^{-i Q \cdot r_{\alpha}(0)} e^{i Q \cdot r_{\alpha}(t)}\right\rangle e^{-i \omega t} d t
\end{aligned}
$$

Pour une espèce chimique donnée, la moyenne $\bar{b}$ des longueurs de diffusion prise sur les différents isotopes et sur tous les états de spin est appelée la longueur de diffusion cohérente, $b^{\text {coh }}$. Dans le même temps, l'écart quadratique moyen de $b$ à $\bar{b}$ définit la longueur de diffusion incohérente:

$$
\begin{aligned}
& b^{c o h}=\bar{b} \\
& b^{i n c}=\sqrt{\bar{b}^{2}-\bar{b}^{2}}
\end{aligned}
$$

Les longueurs de diffusion cohérente et incohérente sont de nature profondément différente. La diffusion cohérente est celle à laquelle le système donnerait lieu si tous les atomes d'une même espèce avaient la même longueur de diffusion $\bar{b}$. Le neutron verrait alors un potentiel uniforme moyen, pouvant donner lieu à des phénomènes d'interférences. La diffusion incohérente est celle qui s'ajoute à la diffusion cohérente pour obtenir la diffusion du système réel. Les écarts au potentiel moyen étant distribués au hasard, ils ne peuvent être à l'origine de phénomènes d'interférences. 
Ces définitions nous permettent d'écrire l'équation précédente sous la forme

$$
\frac{d^{2} \sigma}{d \Omega d \omega}=\left(\frac{d^{2} \sigma}{d \Omega d \omega}\right)_{c o h}+\left(\frac{d^{2} \sigma}{d \Omega d \omega}\right)_{\text {inc }}
$$

On définit habituellement la fonction de diffusion intermédiaire, $F(\boldsymbol{Q}, t)$,

$$
F(\boldsymbol{Q}, t)=\frac{1}{N} \sum_{\alpha} \sum_{\beta}\left\langle. e^{-i \boldsymbol{Q} \cdot \boldsymbol{r}_{\alpha}(0)} e^{i \boldsymbol{Q} \cdot \boldsymbol{r}_{\beta}(t)}\right\rangle
$$

dont on prend les transformées de Fourier suivantes. La transformée de Fourier spatiale, $G(\boldsymbol{r}, t)$ est désignée comme la fonction de corrélation de paires dépendante du temps [7]

$$
G(\boldsymbol{r}, t)=\frac{1}{(2 \pi)^{3}} \int_{-\infty}^{\infty} F(\boldsymbol{Q}, t) e^{-i \boldsymbol{Q} r} d \boldsymbol{Q}
$$

La transformée de Fourier temporelle, $S(\boldsymbol{Q}, \omega)$, est la fonction de diffusion dynamique, (appelée aussi loi de diffusion, ou facteur de structure dynamique)

$$
S(\boldsymbol{Q}, \omega)=\frac{1}{2 \pi} \int_{-\infty}^{\infty} F(\boldsymbol{Q}, t) e^{-i \omega t} d t
$$

D'après les définitions des transformées de Fourier inverses correspondantes

$$
\begin{aligned}
& F(\boldsymbol{Q}, t)=\int_{-\infty}^{\infty} G(\boldsymbol{r}, t) e^{i \boldsymbol{Q} \boldsymbol{r}} d \boldsymbol{r} \\
& F(\boldsymbol{Q}, t)=\int_{-\infty}^{\infty} S(\boldsymbol{Q}, \omega) e^{i \omega t} d t
\end{aligned}
$$

il apparaît que $S(\boldsymbol{Q}, \omega)$ est la transformée de Fourier de $G(\boldsymbol{r}, t)$ par rapport à l'espace et au temps :

$$
\begin{aligned}
& S(\boldsymbol{Q}, \omega)=\frac{1}{2 \pi} \int_{-\infty}^{\infty} G(\boldsymbol{r}, t) e^{-i(\omega t-\boldsymbol{Q} . \boldsymbol{r})} d \boldsymbol{Q} d t \\
& G(\boldsymbol{r}, t)=\frac{1}{(2 \pi)^{3}} \int_{-\infty}^{\infty} S(\boldsymbol{Q}, \omega) e^{i(\omega t-\boldsymbol{Q} . \boldsymbol{r})} d \boldsymbol{Q} d t
\end{aligned}
$$

tandis que $F(\boldsymbol{Q}, t)$ est la transformée de Fourier spatiale de $G(\boldsymbol{r}, t)$ et que $S(\boldsymbol{Q}, \omega)$ est la transformée de Fourier temporelle de $F(\boldsymbol{Q}, t)$.

Lorsque le même diffuseur intervient aux instants 0 et $t$, on définit d'une manière analogue une fonction de diffusion intermédiaire, désignée comme incohérente, $F_{i n c}(\boldsymbol{Q}, t)$ :

$$
F_{\text {inc }}(\boldsymbol{Q}, t)=\frac{1}{N} \sum_{\alpha}\left\langle. e^{-i \boldsymbol{Q} \cdot \boldsymbol{r}_{\alpha}(0)} e^{i \boldsymbol{Q} \cdot \boldsymbol{r}_{\alpha}(t)}\right\rangle
$$

et une fonction de diffusion incohérente, $S_{i n c}(\boldsymbol{Q}, \omega)$ :

$$
S_{i n c}(Q, \omega)=\frac{1}{2 \pi} \int_{-\infty}^{\infty} F_{i n c}(Q, t) e^{-i \omega t} d t
$$

qui est la transformée de Fourier par rapport à l'espace et au temps de la fonction d'autocorrélation de paires dépendante du temps, $G_{s}(r, t)$ :

$$
S_{i n c}(\boldsymbol{Q}, \omega)=\frac{1}{2 \pi} \int_{-\infty}^{\infty} G_{S}(\boldsymbol{r}, t) e^{-i(\omega t-\boldsymbol{Q} \cdot \boldsymbol{r})} d \boldsymbol{Q} d t
$$




$$
G_{s}(\boldsymbol{r}, t)=\frac{1}{(2 \pi)^{3}} \int_{-\infty}^{\infty} S_{i n c}(\boldsymbol{Q}, \omega) e^{i(\omega t-\boldsymbol{Q} \cdot \boldsymbol{r})} d \boldsymbol{Q} d t
$$

Finalement, nous écrirons :

$$
\begin{aligned}
& \frac{d^{2} \sigma}{d \Omega d \omega}=\frac{1}{4 \pi} \frac{k}{k_{0}}\left[\sigma_{c o h} S(Q, \omega)+\sigma_{i n c} S_{i n c}(Q, \omega)\right] \\
& \sigma_{c o h}=4 \pi\left(b^{c o h}\right)^{2} \\
& \sigma_{i n c}=4 \pi\left(b^{i n c}\right)^{2}
\end{aligned}
$$

$\sigma_{c o h}$ et $\sigma_{i n c}$ sont appelées respectivement section efficace de diffusion cohérente et section efficace de diffusion incohérente. Nous signalerons pour terminer qu'en spectrométrie par écho de spins, l'usage est de désigner les fonctions de diffusion intermédiaires $F(\boldsymbol{Q}, t)$ et $F_{\text {inc }}(\boldsymbol{Q}, t)$ par $S(\boldsymbol{Q}, t)$ et $S_{\text {inc }}(\boldsymbol{Q}, t)$ respectivement.

\section{DIFFUSION INCOHERENTE: LES FONCTIONS DE DIFFUSION}

Nous allons maintenant nous intéresser tout particulièrement à la fonction de diffusion intermédiaire incohérente relative à un diffuseur particulier

$$
F_{i n c}(\boldsymbol{Q}, t)=\langle\exp [i \boldsymbol{Q} \cdot \boldsymbol{r}(t)] \cdot \exp [-i \boldsymbol{Q} \cdot \boldsymbol{r}(0)]\rangle
$$

Considérant le cas général d'un composé moléculaire (cristal moléculaire, polymère, liquide moléculaire, ...), nous allons recenser les diverses composantes du vecteur $\boldsymbol{r}(t)$ repérant sa position dans l'échantillon et nous analyserons le caractère de leurs variations au cours du temps sous l'effet des vibrations internes de la molécule ainsi que de ses déplacements d'ensemble. Après avoir précisé les hypothèses selon lesquelles la dynamique de mouvements de nature différente peut être étudiée séparément nous établirons les fonctions de diffusion correspondantes.

\subsection{Séparation des mouvements}

Les variations du vecteur $r(t)$ repérant la position instantanée d'un noyau diffuseur peuvent être décomposées en composantes d'origine différente: les vibrations moléculaires internes et les déplacements d'ensemble de la molécule à laquelle le noyau appartient. Les élongations des liaisons chimiques et les modifications des angles entre ces liaisons ont pour conséquence un déplacement $\boldsymbol{v}(t) \mathrm{du}$ noyau de sa position d'équilibre dans la molécule. Dans le même temps, par rapport à un système d'axes fixes extérieur à la molécule tel celui des axes du réseau dans le cas d'un matériau cristallin, la situation instantanée de cette position d'équilibre varie sous l'effet des déplacements d'ensemble de la molécule. Suivant la nature du matériau, ces derniers peuvent prendre des aspects différents. Les liquides combinent souvent une diffusion à longue distance des molécules avec des rotations ou des réorientations de grande amplitude autour de leurs centres de gravité. Les espèces chimiques incluses en phase solide peuvent aussi être l'objet de déplacements à longue distance, généralement par sauts successifs entre sites d'adsorption. Dans les milieux solides désordonnés on peut observer des réorientations de groupements chimiques comme la réorientation de groupements méthyle latéraux dans des polymères. Le passage entre deux formes énantiomères par l'inversion d'un cycle peut induire des déplacements importants des groupes chimiques qui y sont greffés. Dans les cristaux liquides ou les cristaux plastiques des molécules entières sont susceptibles de se réorienter. Quant aux milieux cristallins, ils sont le siège de mouvements vibrationnels coopératifs (phonons). 
Dans les solides, en absence de diffusion à longue distance, les origines possibles du déplacement d'un atome diffuseur sont les vibrations du réseau cristallin ainsi que les vibrations internes de la molécule dont l'atome fait partie auxquelles il faut ajouter, pour les composés à désordre d'orientation de nature dynamique, les réorientations angulaires de la molécule autour de son centre de gravité. Désignons par $v(t)$ le vecteur correspondant au déplacement d'ensemble de la molécule sous l'effet des vibrations de réseau. De même $\boldsymbol{u}(t)$ tient compte du déplacement de l'atome diffuseur sous l'effet des vibrations moléculaires internes. Enfin, l'orientation de la molécule étant repérée par les angles d'Euler $\Omega$, la position d'équilibre de l'atome dans cette dernière est notée $R(\Omega, t)$. Dès lors la position instantanée de l'atome diffuseur, $\boldsymbol{r}(t)$, s'écrit d'une manière générale:

$$
r(t)=v(t)+\boldsymbol{R}(\boldsymbol{\Omega}, t)+\boldsymbol{u}(t)
$$

Dans les cas de phases liquides ou d'espèces incluses diffusant dans des milieux poreux, on remplacera le vecteur $v(t)$ associé aux vibrations de réseau par un vecteur $\boldsymbol{T}(t)$ représentant la translation de la molécule dans son ensemble:

$$
\boldsymbol{r}(t)=\boldsymbol{T}(t)+\boldsymbol{R}(\boldsymbol{\Omega}, t)+\boldsymbol{u}(t)
$$

Un calcul exact de la fonction de diffusion incohérente qui tienne compte de tous les mouvements que nous venons d'énumérer est très difficile et à notre connaissance n'a jamais été entrepris, pour autant qu'il soit possible.

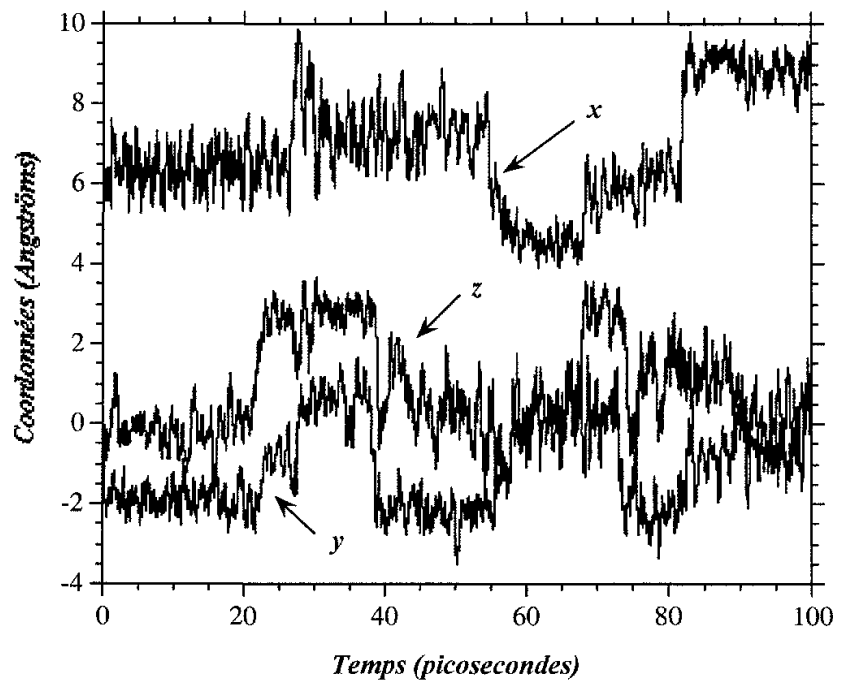

Figure 2: Trajectoire d'un des atomes d'hydrogène de la molécule d'hexaméthyléthane obtenue par simulation de dynamique moléculaire pour une température $T=300 \mathrm{~K}$. Les valeurs des 3 coordonnées $(x, y, z)$ sont représentées sur une durée de 100 picosecondes. La simulation totale dure 660 picosecondes. De brusques changements d'orientation sont clairement visibles. Le temps moyen qui s'écoule entre deux réorientations est de l'ordre de $10^{-11} \mathrm{~s}$. Ce sont ces mouvements qui sont responsables de l'élargissement quasiélastique observé sur les spectres de diffusion de neutrons. Dans chacune de ses orientations d'équilibre la molécule effctue un grand nombre de vibrations et de librations (on notera que les coordonnées ne sont enregistrées que tous les 10 pas de simulation). 
La démarche habituelle consiste à factoriser la fonction de diffusion intermédiaire $F_{\text {inc }}(\boldsymbol{Q}, \boldsymbol{t})$ en plusieurs termes correspondant chacun à un des mouvements que nous venons d'évoquer. Il convient d'examiner les conditions de cette approximation. De notre analyse des diverses composantes du déplacement d'un noyau diffuseur, il est clair que deux échelles de temps sont en cause. Les vibrations internes des molécules sont très rapides $\left(10^{-15}-10^{-14} \mathrm{~s}\right)$ tout comme les vibrations de réseau $\left(10^{-13} \mathrm{~s}\right) \mathrm{si}$ on fait exception des modes acoustiques de très basse fréquence. Les déplacements à longue distance de molécules en phase liquide ou d'espèces incluses sont beaucoup plus lents $\left(10^{-10}-10^{-9} \mathrm{~s}\right)$. Quant aux temps caractéristiques associés aux réorientations de groupements chimiques ou de molécules entières ils sont de l'ordre de $10^{-12}-10^{-11} \mathrm{~s}$

\subsection{Les fonctions de diffusion}

L'hypothèse principale est l'absence de tout couplage entre les mouvements intervenant sur des échelles de temps différentes. La figure 2 montre le résultat d'une simulation de dynamique moléculaire de la molécule d'hexaméthyléthane. Les coordonnées d'un atome d'hydrogène de l'un des groupements méthyles ont été suivies au cours du temps. Les réorientations de la molécule apparaissent clairement sous forme de brutales discontinuités dans la trajectoire. On remarquera aussi le grand nombre d'oscillations effectuées par la molécule sur une position d'équilibre entre deux réorientations successives. Cette observation tend à confirmer la très faible corrélation entre ces deux mouvements. Cette conclusion est renforcée par la comparaison des énergies de vibrations de l'ordre de plusieurs dizaines de meV aux faibles transferts d'énergie ( $<1$ à $2 \mathrm{meV}$ ) associés aux réorientations. De même on néglige habituellement tout couplage entre la translation et la réorientation d'une molécule. Cette seconde hypothèse, pour autant qu'elle soit commode pour les calculs est certainement moins satisfaisante. Elle suppose en particulier que les molécules se réorientent indépendamment les unes des autres et exclue les processus coopératifs. Certains résultats obtenus en dynamique moléculaire tendent à l'infirmer.

On écrira ainsi, pour un solide

$$
\begin{aligned}
& F_{\text {inc }}(\boldsymbol{Q}, t)=\langle\exp (-i \boldsymbol{Q} \cdot \boldsymbol{v}(0)) \cdot \exp (i \boldsymbol{Q} \cdot \boldsymbol{v}(t))\rangle \\
&\langle\exp (-i \boldsymbol{Q} \cdot \boldsymbol{u}(0)) \cdot \exp (i \boldsymbol{Q} \cdot \boldsymbol{u}(t))\rangle \\
&\langle\exp (-i \boldsymbol{Q} \cdot \boldsymbol{R}(\boldsymbol{\Omega}, 0)) \cdot \exp (i \boldsymbol{Q} \cdot \boldsymbol{R}(\boldsymbol{\Omega}, t))\rangle \\
& F_{\text {inc }}(\boldsymbol{Q}, t)=F_{V}(\boldsymbol{Q}, t) \cdot F_{U}(\boldsymbol{Q}, t) \cdot F_{R}(\boldsymbol{Q}, t)
\end{aligned}
$$

et pour un liquide

$$
\begin{gathered}
F_{i n c}(\boldsymbol{Q}, t)=\langle\exp (-i \boldsymbol{Q} \cdot \boldsymbol{T}(0)) \cdot \exp (i \boldsymbol{Q} \cdot \boldsymbol{T}(t))\rangle \\
\langle\exp (-i \boldsymbol{Q} \cdot \boldsymbol{u}(0)) \cdot \exp (i \boldsymbol{Q} \cdot \boldsymbol{u}(t))\rangle \\
\langle\exp (-i \boldsymbol{Q} \cdot \boldsymbol{R}(\boldsymbol{\Omega}, 0)) \cdot \exp (i \boldsymbol{Q} \cdot \boldsymbol{R}(\boldsymbol{\Omega}, t))\rangle \\
F_{i n c}(\boldsymbol{Q}, t)=F_{T}(\boldsymbol{Q}, t) \cdot F_{U}(\boldsymbol{Q}, t) \cdot F_{R}(\boldsymbol{Q}, t)
\end{gathered}
$$

La transformation de Fourier de ces expressions conduit aux fonctions de diffusion correspondantes qui apparaissent comme les produits de convolution des fonctions de diffusion associées à chaque type de mouvement.

$$
\begin{aligned}
& S_{i n c}(\boldsymbol{Q}, \omega)=S_{V}(\boldsymbol{Q}, \omega) \otimes S_{U}(\boldsymbol{Q}, \omega) \otimes S_{R}(\boldsymbol{Q}, \omega) \\
& S_{i n c}(\boldsymbol{Q}, \omega)=S_{T}(\boldsymbol{Q}, \omega) \otimes S_{U}(\boldsymbol{Q}, \omega) \otimes S_{R}(\boldsymbol{Q}, \omega)
\end{aligned}
$$


Il convient d'être prudent dans l'évaluation des moyennes thermiques car les opérateurs mis en jeu ne commutent pas. Moyennant quelques corrections, $S_{T}(\boldsymbol{Q}, \omega)$ et $S_{R}(\boldsymbol{Q}, \omega)$ qui concernent des mouvements lents, de type diffusif, peuvent être évaluées par la mécanique classique. Par contre pour les termes $S_{V}(\boldsymbol{Q}, \omega)$ et $S_{U}(\boldsymbol{Q}, \omega)$ il est absolument nécessaire de faire intervenir la mécanique quantique.

\section{LES VIBRATIONS MOLECULAIRES}

Nous renvoyons aux ouvrages de base [1-5,8,9] pour un calcul détaillé des fonctions de diffusion associées aux mouvements de vibrations intramoléculaires ou aux phonons. Nous nous bornerons à en donner une analyse fort simplifiée

\subsection{La fonction de diffusion vibrationnelle pour un oscillateur harmonique unique}

Considérons le cas d'un atome diffuseur effectuant des mouvements de vibration dont nous ne préciserons pas pour l'instant la nature (interne ou externe). La fonction de diffusion intermédiaire est la moyenne thermique

$$
F(\boldsymbol{Q}, t)=\left\langle\exp U_{0} \cdot \exp U_{t}\right\rangle
$$

où l'on a posé

$$
\begin{aligned}
& U_{0}=-i \boldsymbol{Q} . \boldsymbol{u}(0) \\
& U_{t}=i \boldsymbol{Q} \cdot \boldsymbol{u}(t)
\end{aligned}
$$

Les opérateurs $U_{0}$ et $U_{t}$ ne commutent pas entre eux, mais chacun commute avec leur commutateur $\left[U_{0}, U_{t}\right]$. La relation générale

$$
\exp \left(U_{0}\right) \cdot \exp \left(U_{t}\right)=\exp \left(U_{0}+U_{t}\right) \exp \left(\left[U_{0}, U_{i}\right]\right)
$$

permet d'écrire le produit des deux termes exponentiels sous la forme [3]

$$
F(\boldsymbol{Q}, t)=\exp \left\langle U_{0}^{2}\right\rangle \exp \left\langle U_{0} U_{t}\right\rangle
$$

En effectuant un développement en série limité au second ordre du second facteur exponentiel,

$$
\begin{aligned}
F(\boldsymbol{Q}, t) & =\exp \left(-\left\langle(\boldsymbol{Q} \cdot \boldsymbol{u}(0))^{2}\right\rangle\right) \cdot \exp \langle\boldsymbol{Q} \cdot \boldsymbol{u}(0) \boldsymbol{Q} \cdot \boldsymbol{u}(t)\rangle \\
& =\exp \left(-\left\langle(\boldsymbol{Q} \cdot \boldsymbol{u}(0))^{2}\right\rangle\right) \cdot\left[1+\langle\boldsymbol{Q} \cdot \boldsymbol{u}(0) \boldsymbol{Q} \cdot \boldsymbol{u}(t)\rangle+\frac{1}{2}\langle\boldsymbol{Q} \cdot \boldsymbol{u}(0) \boldsymbol{Q} \cdot \boldsymbol{u}(t)\rangle^{2} \ldots\right]
\end{aligned}
$$

Le premier facteur du membre de droite apparaît comme un terme de Debye-Waller, qui traduit le fait que le phénomène d'interférence entre les ondes neutroniques diffusées par l'atome aux instants 0 et $t$ est atténué par ses propres vibrations thermiques

$$
\begin{aligned}
\exp \left(-\left\langle(Q \cdot u(0))^{2}\right\rangle\right) & =\exp \left(-Q^{2}<u^{2}>\right) \\
& =\exp (-2 W(Q))
\end{aligned}
$$

Le second facteur est un terme purement inélastique faisant apparaître dans son développement la fonction de corrélation du déplacement de l'atome.

Considérons un atome diffuseur soumis à une vibration purement sinusoïdale

$$
\boldsymbol{u}(t)=\boldsymbol{u}_{0} \cos \left(\omega_{0} t\right)
$$

En négligeant les termes d'ordre supérieur dans le développement (35) la fonction de diffusion intermédiaire s'évalue comme 


$$
F(\boldsymbol{Q}, t)=\exp (-2 W(Q)) \cdot\left[1+\left\langle\left(\boldsymbol{Q} \cdot \boldsymbol{u}_{0}\right)^{2}\right\rangle \cos \left(\omega_{0} t\right)\right]
$$

Par transformation de Fourier, la fonction cosinus introduit dans la fonction de diffusion dynamique deux pics de Dirac, centrés respectivement en $\omega_{0}$ et $-\omega_{0}$, tandis que le terme indépendant du temps donne un autre pic de Dirac centré en $\omega=0$ selon

$$
S(Q, \omega)=\exp (-2 W(Q)) \cdot\left[\delta(\omega)+\frac{\left\langle\left(\boldsymbol{Q} \cdot \boldsymbol{u}_{0}\right)^{2}\right\rangle}{2}\left\{\delta\left(\omega+\omega_{0}\right)+\delta\left(\omega-\omega_{0}\right)\right\}\right]
$$

La première fonction de Dirac correspond à une diffusion élastique sans changement d'énergie des neutrons, les deux autres correspondent soit à l'absorption soit à l'émission d'un phonon qui s'ajoute ou se soustrait à l'énergie incidente. Dans la pratique les fonctions de Dirac sont élargies par la résolution instrumentale et le spectre prend l'allure de la figure 3.

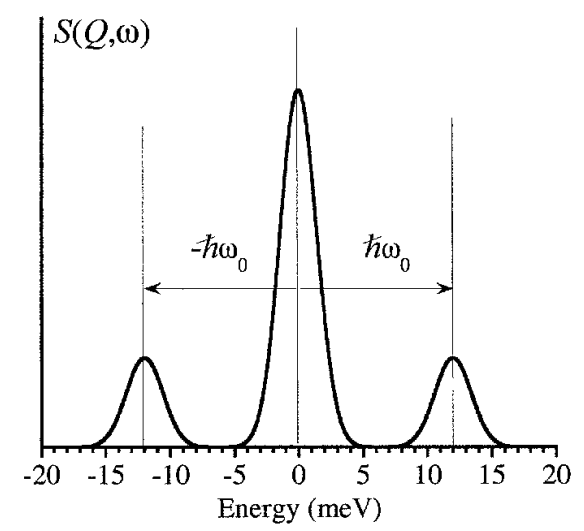

Figure 3: Représentation schématique de la fonction de diffusion pour un oscillateur simple

Comme nous avons considéré le cas d'un oscillateur en mécanique classique, les intensités sont les mêmes en gain comme en perte d'énergie, et la condition du bilan détaillé n'est pas respectée. Les termes inélastiques contiennent un facteur de polarisation $\left(\boldsymbol{Q} . \boldsymbol{u}_{0}\right)^{2}$ qui indique que la diffusion inélastique est maximale pour un transfert de moment parallèle à la direction du déplacement de l'atome et nulle quand il est perpendiculaire. Pour un angle donné entre $\boldsymbol{Q}$ et $\boldsymbol{u}_{0}$ l'intensité inélastique est d'autre part proportionnelle à $Q^{2}=\left(16 \cdot \pi^{2} \cdot \sin ^{2} \theta\right) / \lambda^{2}, 2 \theta$ étant l'angle de diffusion.

En portant l'expression du déplacement de l'atome dans celle de la fonction intermédiaire, et en tenant compte de son énergie cinétique moyenne

$$
\frac{1}{2} M\left\langle\left(\frac{d u}{d t}\right)^{2}\right\rangle=\frac{M u_{0}^{2} \omega_{0}^{2}}{4}=\frac{1}{2} k_{B} T
$$

il est possible de mettre cette fonction sous la forme

$$
F(\boldsymbol{Q}, t)=\exp \left\{-\frac{Q^{2} \sigma^{2}(t)}{2}\right\}
$$

avec

$$
\sigma^{2}(t)=\frac{2 k_{B} T}{M \omega_{0}{ }^{2}}\left(1-\cos \omega_{0} t\right)
$$

La transformée de Fourier spatiale fournit la fonction de corrélation correspondante 


$$
G_{s}(r, t)=\frac{1}{\left[2 \pi \sigma^{2}(t)\right]^{3 / 2}} \exp \left\{-\frac{r^{2}}{2 \sigma^{2}(t)}\right\}
$$

qui présente une dépendance gaussienne en $r$. Ainsi le comportement de $G_{s}(r, t)$ est complètement déterminé par une fonction $\sigma^{2}(t)$ qui représente en réalité le déplacement carré moyen pendant l'intervalle de temps $t$.

\subsection{La fonction de diffusion vibrationnelle pour une molécule}

Supposons que l'atome considéré appartienne à une molécule formée au total de $N$ atomes. La théorie des vibrations moléculaires décrit les déplacements des atomes de la molécule comme résultant du couplage de $3 N-6$ oscillateurs harmoniques, associés chacun à une fréquence bien déterminée (modes propres). La fonction de diffusion vibrationnelle, résultant des déplacements de tous les atomes se déduit de la généralisation de la figure précédente en remplaçant chacun des deux pics inélastiques par un ensemble de $3 N-6$ pics de Dirac correspondant aux modes de vibration propres de la molécule.

La figure 4 montre l'exemple d'un spectre inélastique obtenu avec un échantillon biologique à basse température sur le spectromètre TFXA du Rutherford-Appleton Laboratory (Royaume-Uni).

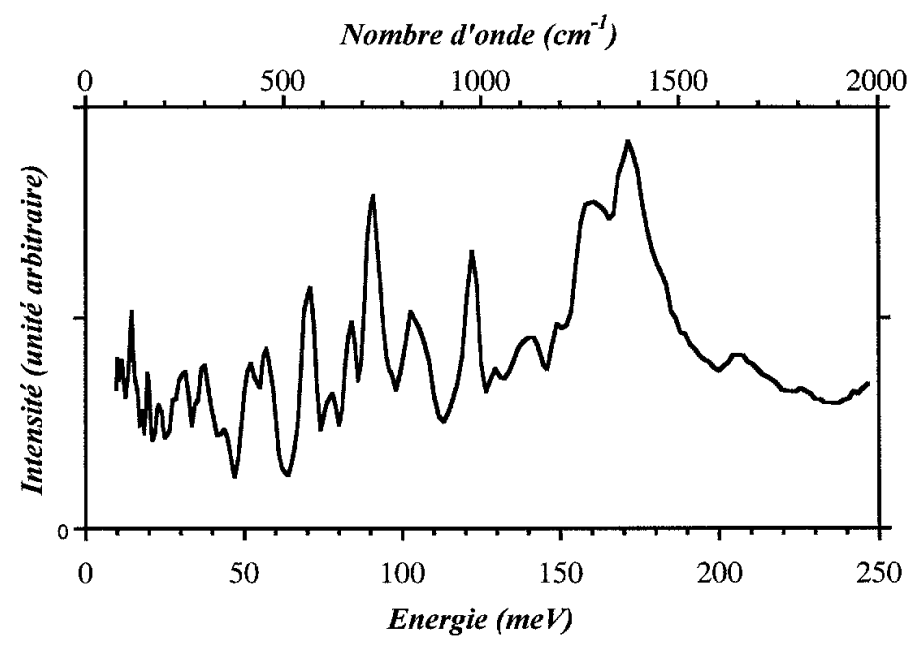

Figure 4: Spectre inélastique de la cytidine obtenu à $T=15 \mathrm{~K}$ avec le spectromètre TFXA du Rutherford-Appleton Laboratory (reproduit avec la permission de [10]).

Le spectromètre TXFA (Time Focused Crystal Analyser) était un instrument à géométrie inversée, spécialement conçu pour la spectroscopie moléculaire dans la région $2-500 \mathrm{meV}\left(16-4000 \mathrm{~cm}^{-1}\right)$. L'échantillon recevait des bouffées polychromatiques de neutrons. Une partie des neutrons qui avaient une énergie suffisante pour exciter des transitions internes dans l'échantillon étaient reçus par un système de détection, placé à un angle de diffusion de $135^{\circ}$. Celui-ci était constitué successivement d'un graphite pyrolytique (002) qui sélectionnait les neutrons d'énergie finale de $3 \mathrm{meV}$, d'un filtre au béryllium éliminait les réflexions de Bragg d'ordre supérieur et de 14 tubes à ${ }^{3} \mathrm{He}$. En raison de la faible énergie finale des neutrons, le vecteur de diffusion était pratiquement parallèle à la direction du faisceau incident, ce qui permettait l'étude d'échantillons orientés. TFXA a été remplacé par TOSCA en 1998, qui offre une plus grande sensibilité (deux groupes de détecteurs) et une meilleure résolution en énergie.

Le spectromètre IN1-BeF installé à l'ILL utilise un jeu de monochromateurs pour faire varier l'énergie des neutrons incidents et un filtre au béryllium pour déterminer leur énergie finale. Il couvre une gamma 
d'énergie de $16 \mathrm{meV}$ à $800 \mathrm{meV}$. Quant au spectromètre à temps de vol IN4 il couvre le domaine 10-100 $\mathrm{meV}$. L'analyse de la région basse fréquence du spectre peut être réalisée sur des spectromètres de type temps de vol utilisant des neutrons froids (IN5 et IN6 à l'ILL, MIBEMOL au LLB). Contrairement aux spectromètres précédents, ces instruments fonctionnent en gain d'énergie du neutron et permettent d'étudier des échantillons autour de la température ambiante. La figure 8 représente un tel exemple, que nous commenterons plus loin.

\subsection{Diffusion par les phonons}

En passant d'un ensemble atomique constituant une molécule à celui d'un réseau cristallin, le nombre d'atomes augmente considérablement. Si les déplacements des atomes autour de leurs positions d'équilibre restent petits comparés aux distances séparant ces dernières, un cristal peut être modélisé comme un ensemble d'oscillateurs harmoniques couplés. Compte tenu du nombre considérable d'atomes en interaction, on utilise la symétrie de translation du réseau: les déplacement atomiques sont décrits comme une superposition d'ondes planes, les modes de phonons, associés chacun à une fréquence caractéristique. L'étude de la dynamique des réseaux cristallins devient très vite compliquée pour peu que l'on tienne compte de diverses interactions entre les atomes. Nous nous bornerons ici au cas d'une chaîne linéaire formée de deux types d'atomes différents, de masses respectives $M_{1}$ et $M_{2}$, séparés par une distance $a$.

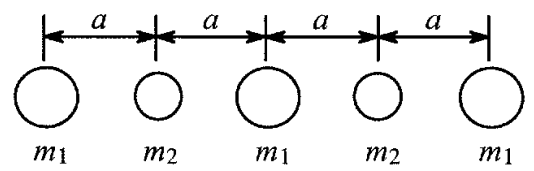

Figure 5: Représentation schématique de la chaine linéaire formée de deux types d'atomes différents.

Les équations de la dynamique montrent que la fréquence d'un mode de phonon est liée à son vecteur d'onde, $q$, par l'expression

$$
\omega_{ \pm}^{2}=\frac{C}{\mu}\left[1 \pm \sqrt{1-\frac{4 \mu}{M} \sin ^{2} q a}\right]
$$

dans laquelle $C$ est la constante d'interaction, $M=M_{1}+M_{2}$ et $\mu$ est la masse réduite

$$
\mu=\frac{M_{1} M_{2}}{M_{1}+M_{2}}
$$

La solution $\omega .(q)$ est telle que

$$
\lim _{q \rightarrow 0} \omega_{-}^{2}(q)=\frac{2 C a^{2}}{M} q^{2}
$$

Et s'annule pour $q=0$, les déplacements des atomes devenant identiques comme dans les vibrations acoustiques de grande longueur d'onde: cette courbe porte le nom de branche acoustique. Sa tangente est horizontale en limite de zone de Brillouin $(q a=\pi)$. L'autre solution admet aussi une tangente horizontale en limite de zone, mais aussi en centre de zone, avec des vibrations des deux types d'atomes en opposition de phase: c'est la branche optique.

Ces modes de vibration qui font intervenir des corrélations entre les déplacements d'atomes différents donnent lieu à une diffusion cohérente des neutrons. Le calcul de la fonction de diffusion cohérente fait apparaître dans le processus de création ou d'annihilation d'un phonon deux fonctions de Dirac. La première assure la conservation de l'énergie du système neutron-phonon, l'autre celle du vecteur d'onde: en raison de la symétrie de translation, ces processus ne peuvent se produire que si le transfert de moment du neutron, $\boldsymbol{Q}$, est tel que 


$$
Q=K \pm q
$$

$K$ étant un vecteur du réseau réciproque. La diffusion cohérente permet de mesurer point par point les courbes de dispersion $\omega(\boldsymbol{q})$.

On utilise pour cela un spectromètre à trois axes. Ce type d'instrument a été inventé par Brockhouse en 1962. Il permet d'étudier des excitations bien définies comme les phonons ou les ondes de spin dans des monocristaux. Depuis son apparition ces spectromètres ont peu changé et ils peuvent être schématisés comme le montre la figure 6 . Les deux vecteurs d'onde initial $\boldsymbol{k}_{\boldsymbol{i}}$ et final $\boldsymbol{k}_{\boldsymbol{f}}$ sont sélectionnés par des réflexions de Bragg sur des cristaux. Le réglage de l'orientation de l'échantillon permet de positionner à volonté le transfert de moment dans toutes les directions du réseau réciproque. Plusieurs modes d'analyse permettent d'obtenir la meilleure résolution possible en énergie ou en vecteur d'onde. Le mode à $\boldsymbol{Q}$ constant, consiste à maintenir le transfert de moment selon la relation (47). Dès lors il est possible de fixer l'énergie initiale et de faire varier l'énergie finale, ou inversement.

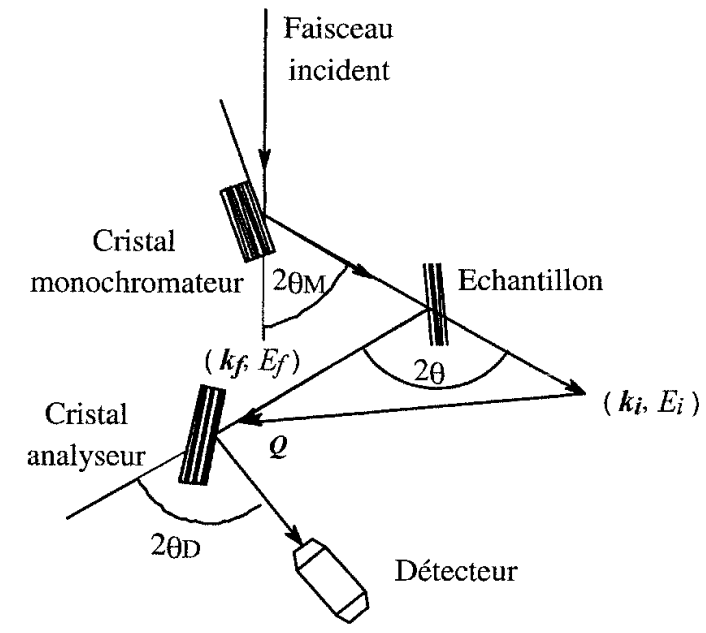

Figure 6: Représentation schématique d'un spectromètre trois axes.

La dynamique des matériaux amorphes ou vitreux se situe entre celle des liquides et celle des solides et montre des oscillations très fortement amorties des atomes autour de sites de pseudo équilibre. Ces excitations ont une durée de vie longue si on les compare à leur période d'oscillation, mais suffisamment courte pour que les pics de phonons qui leur correspondent soient très larges.

La fonction de diffusion incohérente ne présente pas de loi de conservation du moment. Elle est cependant intéressante car elle permet de mesurer la distribution des fréquences de vibrations, $g(\omega)$. Cette dernière est définie comme

$$
g(\omega)=\frac{1}{6 V^{*}} \sum_{q j} \delta\left[\omega-\omega_{j}(q)\right]
$$

$V^{*}$ est le volume de la maille du réseau réciproque. La somme porte sur toutes les branches, $j$ et sur tous les vecteurs d'onde de la première zone de Brillouin. La fonction de distribution des fréquences est normalisée 


$$
\int_{0}^{\infty} g(\omega) d \omega=1
$$

Pour un réseau cubique, comprenant un seul type d'atome, la fonction de diffusion incohérente s'exprime sous la forme:

avec le facteur de Debye-Waller

$$
S_{U}(Q, \omega)=\exp [-2 W(Q)]\left[\delta(\omega)+\frac{\hbar Q^{2}}{4 M \omega} \frac{\exp \left(-\frac{\hbar \omega}{2 k_{B} T}\right)}{\sinh \left(\frac{\hbar \omega}{2 k_{B} T}\right)} g(\omega)\right]
$$

$$
2 W(Q)=\frac{\hbar^{2} Q^{2}}{2 M} \int \frac{g(\omega)}{\omega} \operatorname{coth}\left(\frac{\hbar \omega}{2 k_{B} T}\right) d \omega
$$

Cette expression ne tient pas compte des termes multiphonons. La fonction de Dirac correspond à la diffusion élastique. On peut ainsi obtenir directement la fonction de distribution des fréquences, sous réserve d'utiliser dans un premier temps une forme approchée de $g(\omega)$, comme par exemple un spectre de Debye, pour évaluer le facteur de Debye-Waller dans lequel cette distribution intervient explicitement. Cela suppose aussi que les contributions multiphonons sont faibles et puissent être négligées. Dans la pratique expérimentale, ces effets sont minimisés en effectuant les mesures si possible à basse température (donc en perte d'énergie du neutron) et pour de faibles valeurs du transfert de moment (petits angles et faible énergie incidente). Pour traiter ce problème, des méthodes ont été développées. Une procédure itérative est nécessaire. Cependant les processus à plusieurs phonons n'apportent généralement pas d'information utile car ils conduisent souvent à un spectre inélastique relativement peu structuré. Ainsi, même si leur contribution est appréciable dans l'intensité diffusée, l'observation des transitions à un seul phonon reste encore possible.

La fonction $g(\omega)$ contient un certain nombre de singularités lorsque

$$
\operatorname{grad}_{q} \omega_{j}(q)=0
$$

c'est-à-dire pour les fréquences des phonons correspondant aux branches acoustiques et optiques en limite de zone de Brillouin et pour les branches optiques en centre de zone (figure 7). De grands maxima apparaissent dans l'intensité diffusée qui donnent une grossière estimation des courbes de dispersion.

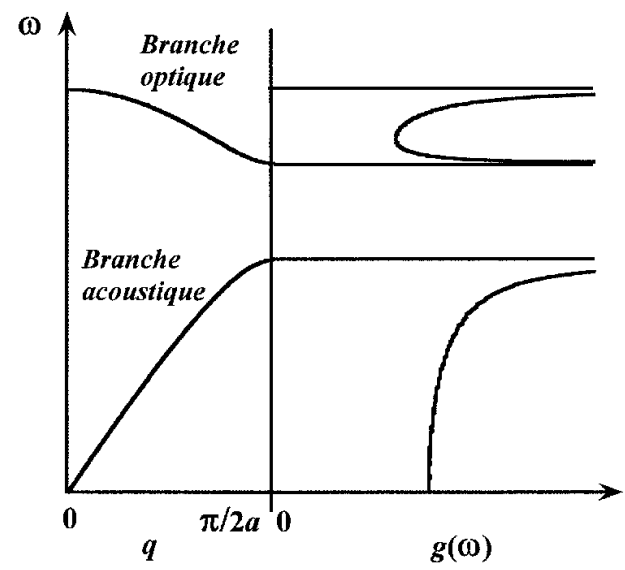

Figure 7: Courbes de dispersion de fréquences et densité d'états de vibration correspondante pour la chaine linéaire composée de deux types d'atomes. 
En réalité l'expression (51) n'est valable que pour les réseaux cubiques monoatomiques. Dans la majorité des cas, et avec des échantillons polycristallins, on doit se contenter d'une distribution des fréquences effective, $G(\omega)$ dans laquelle les différents modes de phonons sont pondérés par le carré de leurs vecteurs polarisation. Même si la relation entre $G(\omega)$ et $g(\omega)$ n'est pas évidente, les maxima de l'intensité diffusée signalés précédemment subsistent et permettent d'avoir une idée des courbes de dispersion quand il est impossible de disposer de monocristaux (à basse température par exemple) ou quand la section efficace cohérente est trop faible. Enfin, avec des échantillons anisotropes (films, fibres,...) des études en fonction de l'orientation de $\boldsymbol{Q}$ permettront d'analyser la polarisation des modes de phonons observables.

En posant

$$
\begin{aligned}
& \bar{\alpha}=\frac{\hbar^{2} Q^{2}}{2 M k_{B} T} \\
& \bar{\beta}=\frac{\hbar \omega}{k_{B} T}
\end{aligned}
$$

il est possible de définir à partir de la partie inélastique de la fonction de diffusion incohérente

une fonction

$$
S_{U}^{I}(Q, \omega)=\exp [-2 W(Q)] \frac{\hbar Q^{2}}{4 M \omega} \frac{\exp \left(-\frac{\hbar \omega}{2 k_{B} T}\right)}{\sinh \left(\frac{\hbar \omega}{2 k_{B} T}\right)} G(\omega)
$$

$$
\begin{aligned}
p(\bar{\alpha}, \bar{\beta}) & =\bar{\beta}[\exp (\bar{\beta})-1] \frac{S_{t z}^{\prime}(Q, \omega)}{\bar{\alpha}} \\
& =\exp [-2 W(Q)] G(\omega)
\end{aligned}
$$

qui, lorsqu'elle est extrapolée à l'origine des vecteurs d'onde, permet de déterminer la fonction de distribution de fréquences $G(\omega)$.

$$
\lim _{Q \rightarrow 0} p(\bar{\alpha}, \bar{\beta})=G(\omega)
$$

La figure 8 montre la fonction $p(\bar{\alpha}, \bar{\beta})$ déduite des spectres de diffusion inélastique de l'acide camphre sulfonique mesurés avec le spectromètre IN6 à l'ILL [11]. Ils ont été obtenus en gain d'énergie du neutron de sorte que l'on observe une forte augmentation de l'intensité avec la température. Ces spectres ne sont pas corrigés des effets multiphonons qui sont particulièrement importants aux plus grandes valeurs du transfert de moment. Mais la résolution du spectromètre se dégrade assez rapidement avec le transfert d'énergie et cette contribution perd rapidement ses pics caractéristiques éventuels. Les pics que l'on observe distinctement entre $10 \mathrm{meV}$ et $40 \mathrm{meV}$ correspondent à des vibrations internes. Des calculs récents ont montré qu'ils correspondaient à des torsions des groupements méthyles. Par exemple les pics à 11 et $21 \mathrm{meV}$ ont pour origine principale respective des torsions en phase et en opposition de phase des deux methyles de la molecule. Les autres raies à 15 et $19 \mathrm{meV}$ s'expliquent par la rotation d'un seul groupement méthyle couplé à une déformation de la cage moléculaire. Les raies observées au dessous de $10 \mathrm{meV}$ ont essentiellement pour origine les modes externes (modes de phonons) de la molécule. 

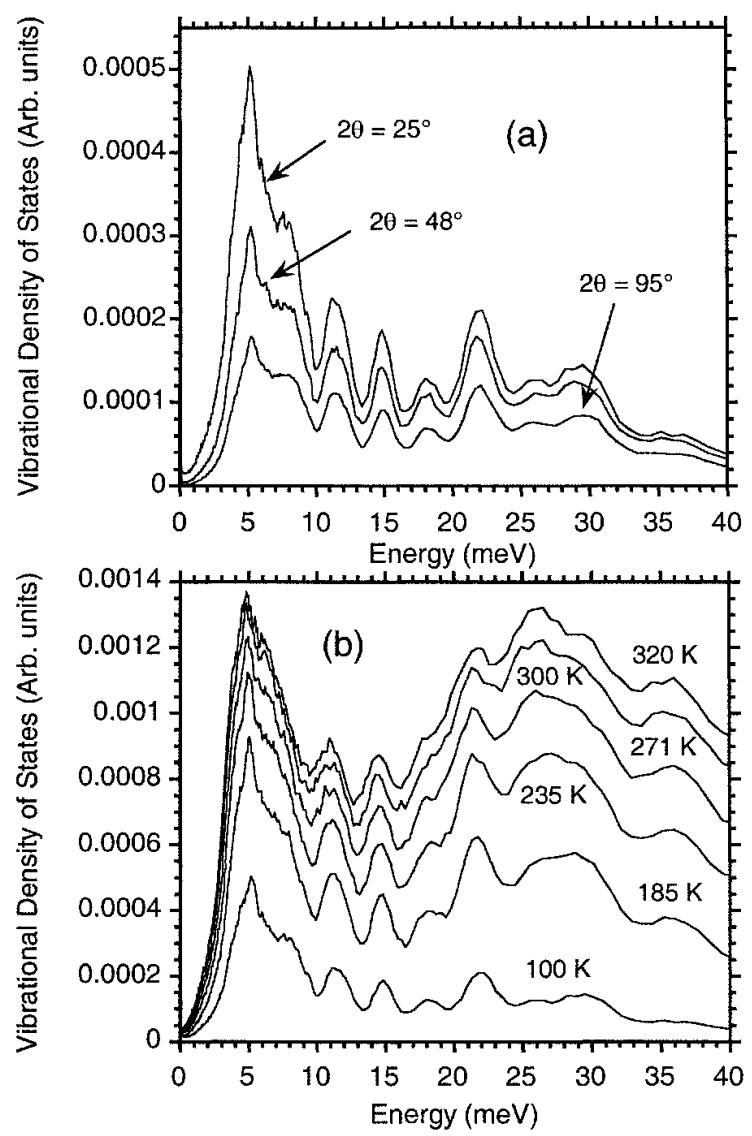

Figure 8: Spectres inélastiques obtenus avec l'acide camphre sulfonique sur le spectromètre IN6 (ILL) (a) Les données enregistrées à $T=100 \mathrm{~K}$ ont été regroupées en trois spectres correspondant (de haut de bas) à des angles moyens de diffusion $25^{\circ}, 48^{\circ}$ et $95^{\circ}$. (b) Evolution avec la température du même spectre correspondant à un angle moyen de diffusion $2 \theta=25^{\circ}(T=$ $100 \mathrm{~K}, 185 \mathrm{~K}, 235 \mathrm{~K}, 271 \mathrm{~K}, 300 \mathrm{~K}$ et $320 \mathrm{~K}$, de bas en haut).

\subsection{Caractères communs aux fonctions de diffusion vibrationnelles}

Il ressort de notre analyse que chacune des fonctions de diffusion associée aux vibrations moléculaires et aux phonons peut être décomposée formellement en une partie élastique et une partie inélastique

$$
\begin{aligned}
& S_{V}(\boldsymbol{Q}, \omega)=\exp \left(-2 W_{V}(Q)\right)\left\{\delta(\omega)+S_{V}^{\mathrm{inbl}}(\boldsymbol{Q}, \omega)\right\} \\
& S_{U}(\boldsymbol{Q}, \omega)=\exp \left(-2 W_{U}(Q)\right)\left\{\delta(\omega)+S_{V}^{\text {inel }}(\boldsymbol{Q}, \omega)\right\}
\end{aligned}
$$

Ces expressions font apparaitre des termes de Debye-Waller 


$$
\begin{aligned}
& 2 W_{V}(Q)=<{ }^{2}>Q^{2} \\
& 2 W_{u}(Q)=<u^{2}>Q^{2}
\end{aligned}
$$

dans lesquels $\left\langle{ }^{2}\right\rangle$ et $\left\langle u^{2}\right\rangle$ représentent les déplacements carrés moyens du diffuseur sous l'effet respectivement des vibrations internes et des vibrations et librations d'ensemble de la molécule. $S_{V}^{\text {inel }}(\boldsymbol{Q}, \omega)$ se compose d'un ensemble de raies étroites (pics de Dirac) caractéristiques des modes normaux de la molécule. Bien que ces raies s'élargissent quand elles sont convoluées avec les autres termes, en raison de leur localisation aux transferts d'énergie élevés, elles n'ont aucune incidence sur la partie quasiélastique des spectres. La fonction $S_{U j}^{\text {inél }}(\boldsymbol{Q}, \omega)$ est liée à la densité des états de vibration du réseau. Elle contribue à l'intensité diffusée dans la région des faibles transferts d'énergies jusqu'à environ $20 \mathrm{meV}$. Elle présente des maxima pour les fréquences optiques situées en centre de zone de Brillouin et pour les modes optiques et acoustiques en limite de zone. Cependant, dans la région quasiélastique cette intensité est généralement de peu d'importance et prend la forme d'un bruit de fond pratiquement plat (figure 9)

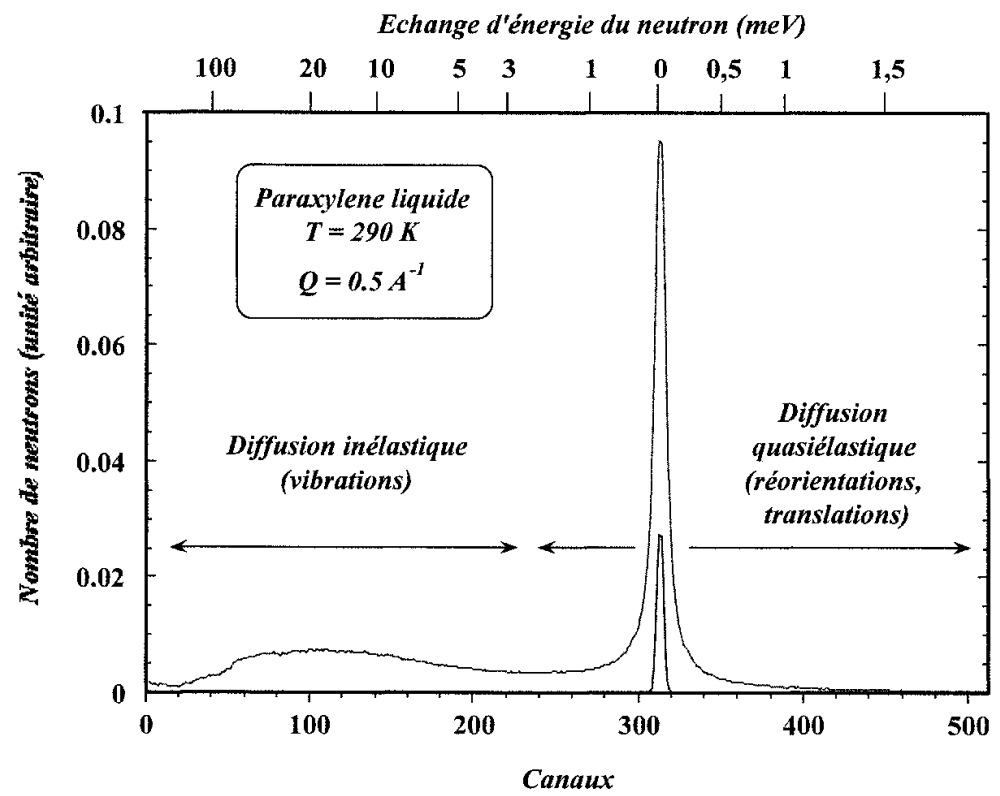

Figure 9 : Exemple de spectre de diffusion quasiélastique obtenu sur un spectromètre du type «temps de vol ». L'échelle en canaux (donc en temps) montre clairement la séparation en une région inélastique correspondant aux modes de vibrations et une région quasiélastique associées aux réorientations et aux translations. La fonction de résolution expérimentale esr représentée et permet de se rendre compte de l'élargissement du pic quasiélastique. forme

Ces considérations nous autorisent à écrire le résultat des produits de convolutions précédents sous la

$$
S_{i n c}(\boldsymbol{Q}, \omega)=\exp (-2 W(Q))\left\{S_{R}(\boldsymbol{Q}, \omega)+S^{i n e ́ l}(\boldsymbol{Q}, \omega)\right\}
$$

pour un solide et 


$$
S_{i n c}(\boldsymbol{Q}, \omega)=\exp (-2 W(Q))\left\{S_{T}(\boldsymbol{Q}, \omega) \otimes S_{R}(\boldsymbol{Q}, \omega)+S^{i n e ́ l}(\boldsymbol{Q}, \omega)\right\}
$$

en présence d'un désordre de translation. Le terme de Debye-Waller

$$
W(Q)=W_{V}(Q)+W_{U}(Q)
$$

tient compte de façon globale des vibrations intramoléculaires et des vibrations de réseau. Le terme inélastique global $S^{\text {inél }}(\boldsymbol{Q}, \omega)$ résulte de la convolution de $S_{V}(\boldsymbol{Q}, \omega)$ et $S_{U}(\boldsymbol{Q}, \omega)$ avec $S_{R}(\boldsymbol{Q}, \omega)$ ou de $S_{U}(\boldsymbol{Q}, \omega)$ avec $S_{T}(\boldsymbol{Q}, \omega) \otimes S_{R}(\boldsymbol{Q}, \omega)$. Il ne concerne qu'une très faible part de l'intensité diffusée dans la région quasiélastique et peut toujours être pris en compte sous la forme d'une densité d'état de Debye.

$S_{T}(\boldsymbol{Q}, \omega)$ et $S_{R}(\boldsymbol{Q}, \omega)$ sont les grandeurs fondamentales qui seront étudiées ici. Ce sont les termes quasiélastiques dont l'analyse renseigne sur les mouvements diffusifs des atomes de l'échantillon.

\subsection{Calcul des fonctions de diffusion dans l'approximation classique}

Comme nous l'avons mentionné les fonctions de diffusion rotationnelle et translationnelle de calculent par la mécanique classique. En effet les échanges d'énergie $\hbar \omega$ ainsi que les transferts de moment $\hbar Q$ mis en jeu sont tels que

$$
\begin{aligned}
& |\hbar \omega|<<\frac{1}{2} k_{B} T \\
& \frac{\hbar^{2} Q^{2}}{2 M} \ll \frac{1}{2} k_{B} T
\end{aligned}
$$

où $M$ est la masse du diffuseur et $\frac{1}{2} k_{B} T$ correspond à l'énergie thermique par degré de liberté. Dans ces conditions $\boldsymbol{R}(\boldsymbol{\Omega}, t)$ ou $\boldsymbol{T}(t), \boldsymbol{R}(\Omega, 0)$ ou $\boldsymbol{T}(0)$ sont simplement des vecteurs $\boldsymbol{R}(t)$ et $\boldsymbol{R}(0)$ repérant les positions du noyau diffuseur aux instants respectifs $t$ et 0 . Le calcul de la fonction intermédiaire se ramène alors à un calcul de probabilités

$$
F_{i n c}(\boldsymbol{Q}, t)=\iint p(\boldsymbol{R}(0) ; \boldsymbol{R}(t)) p(\boldsymbol{R}(0)) \exp (-i \boldsymbol{Q} \cdot \boldsymbol{R}(0)) \exp (i \boldsymbol{Q} \cdot \boldsymbol{R}(t)) d \boldsymbol{R}(t) d \boldsymbol{R}(0)
$$

dans lequel $p(\boldsymbol{R}(0) ; \boldsymbol{R}(t))$ représente la probabilité qu'un atome diffuseur, situé en $\boldsymbol{R}(0)$ à l'instant initial, soit en $\boldsymbol{R}(t)$ à l'instant $t$. La distribution des probabilités à l'instant initial est décrite par $p(\boldsymbol{R}(0))$. Ces fonctions caractérisent le modèle utilisé pour décrire la dynamique de l'échantillon. En général elles dépendent de paramètres physiques (longueurs de sauts, temps caractéristiques...) qui sont déterminés par comparaison et affinement de la loi de diffusion théorique aux spectres expérimentaux.

Les lois de diffusion calculées théoriquement sont symétriques

$$
S^{c l}(\boldsymbol{Q}, \omega)=S^{c l}(-\boldsymbol{Q},-\omega)
$$

et violent le principe du bilan détaillé

$$
S(\boldsymbol{Q}, \omega)=\exp \left(\frac{\hbar \omega}{k_{B} T}\right) S(-\boldsymbol{Q},-\omega)
$$




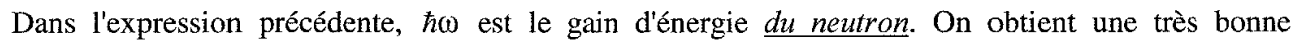
approximation de la fonction de diffusion réelle en posant

$$
S(\boldsymbol{Q}, \omega)=\exp \left(-\frac{\hbar \omega}{2 k_{B} T}\right) S^{c l}(\boldsymbol{Q}, \omega)
$$

\section{PROPRIETES GENERALES DES FONCTIONS DE DIFFUSION ROTATIONNELLES ET TRANSLATIONNELLES}

Nous allons nous intéresser aux expressions générales des fonctions de diffusion associées aux mouvements de rotation et de translation. Reprenons l'expression de la fonction de diffusion intermédiaire relative à un diffuseur unique

$$
F(\boldsymbol{Q}, t)=\langle\exp [i \boldsymbol{Q} \cdot \boldsymbol{r}(t)] \cdot \exp [-i \boldsymbol{Q} \cdot \boldsymbol{r}(0)]\rangle
$$

$F(\boldsymbol{Q}, t)$ désigne indifféremment $F_{T}(\boldsymbol{Q}, t)$ ou $F_{R}(\boldsymbol{Q}, t)$. De même les vecteurs $\boldsymbol{r}(0)$ et $\boldsymbol{r}(t)$ désignent respectivement les vecteurs $T(0)$ et $\boldsymbol{R}(\Omega, 0)$ ou $T(t)$ et $\boldsymbol{R}(\Omega, t)$. Les crochets indiquent une moyenne thermique à prendre à la fois sur les positions à l'instant initial et sur celles à l'instant $t$. Pour des mouvements de type diffusifs cette moyenne peut s'évaluer classiquement de la manière suivante:

$$
F(\boldsymbol{Q}, t)=\iint p(\boldsymbol{R}(0) ; \boldsymbol{R}(t)) p(\boldsymbol{R}(0)) \exp (-i \boldsymbol{Q} \cdot \boldsymbol{R}(0)) \exp (i \boldsymbol{Q} \cdot \boldsymbol{R}(t)) d \boldsymbol{R}(t) d \boldsymbol{R}(0) .
$$

Dans cette expression le terme $p(\boldsymbol{R}(0) ; \boldsymbol{R}(t))$ désigne la probabilité conditionnelle, pour que l'atome diffuseur soit à l'instant $t$ au point de coordonnées $\boldsymbol{R}(t)$, compte tenu d'une position $\boldsymbol{R}(0)$ à l'instant initial. L'occupation de cette position initiale $\boldsymbol{R}(0)$ est soumise à la probabilité $p(\boldsymbol{R}(0))$. Cette dernière est une probabilité liée à un système en équilibre et peut être connue sans difficulté majeure. Par exemple on accordera la même probabilité de $1 / 3$ à l'occupation de chacun des 3 sites possibles pour chaque atome d'hydrogène d'un groupement méthyle susceptible de se réorienter par des sauts de $120^{\circ}$ autour de son axe de symétrie ternaire. Par contre la probabilité conditionnelle $p(\boldsymbol{R}(0) ; \boldsymbol{R}(t))$ ne peut s'obtenir qu'au travers d'un modèle dynamique qui nécessite dans les cas les plus simples, la résolution d'un système d'équations différentielles du premier ordre.

Les corrélations entre les positions du même diffuseur à l'instant initial $\boldsymbol{R}(0)$ et à un instant ultérieur, $\boldsymbol{R}(t)$, diminuent progressivement au cours du temps et finissent par disparaître complètement au temps infini. La probabilité conditionnelle devient indépendante de la position initiale et se confond avec la distribution de probabilité d'équilibre

$$
\lim _{t \rightarrow \infty} p(\boldsymbol{R}(0) ; \boldsymbol{R}(t))=p(\boldsymbol{R}(0) ; \boldsymbol{R}(\infty))=p(\boldsymbol{R}(\infty))
$$

Ainsi la moyenne précédente peut-elle être prise séparément sur les positions initiale et finale du diffuseur

$$
\begin{aligned}
F(\boldsymbol{Q}, \infty) & =\iint p(\boldsymbol{R}(\infty)) p(\boldsymbol{R}(0)) \exp (-i \boldsymbol{Q} \cdot \boldsymbol{R}(0)) \exp (i \boldsymbol{Q} \cdot \boldsymbol{R}(\infty)) d \boldsymbol{R}(\infty) d \boldsymbol{R}(0) \\
& =\left\{\int p(\boldsymbol{R}(0)) \exp (-i \boldsymbol{Q} \cdot \boldsymbol{R}(0)) d \boldsymbol{R}(0)\right\} \cdot\left\{\int p(\boldsymbol{R}(\infty)) \exp (i \boldsymbol{Q} \cdot \boldsymbol{R}(\infty)) d \boldsymbol{R}(\infty)\right\}
\end{aligned}
$$

Puisque le système est en équilibre, la distribution des noyaux diffuseurs est la même à l'instant initial et au temps infini, de sorte que

$$
F(\boldsymbol{Q}, \infty)=\left|\int p(\boldsymbol{R}(0)) \exp (-i \boldsymbol{Q} \cdot \boldsymbol{R}(0)) d \boldsymbol{R}(0)\right|^{2}=\left|\int p(\boldsymbol{R}(\infty)) \exp (i \boldsymbol{Q} \cdot \boldsymbol{R}(\infty)) d \boldsymbol{R}(\infty)\right|^{2}
$$




\section{NEUTRONS ET SYSTÈMES DÉSORDONNÉS}

La limite précédente de la fonction de diffusion intermédiaire est d'une importance considérable en diffusion quasiélastique et sa valeur a des conséquences directement observables dans la forme des spectres obtenus. Elle s'exprime comme le carré de la transformée de Fourier de la distribution d'équilibre des positions accessibles à un noyau diffuseur.

A un instant quelconque, il est possible de séparer formellement $F(Q, t)$ en sa partie indépendante du temps, $F(\boldsymbol{Q}, \infty)$ et une partie dépendante du temps $F_{1}(\boldsymbol{Q}, t)$

$$
F(\boldsymbol{Q}, t)=F(\boldsymbol{Q}, \infty)+F_{1}(\boldsymbol{Q}, t)
$$

En prenant la transformée de Fourier, la présence du terme constant, $F(\boldsymbol{Q}, \infty)$ fait apparaître dans l'expression de la fonction de diffusion incohérente une composante purement élastique $F(\boldsymbol{Q}, \infty) . \delta(\omega)$

$$
\begin{aligned}
S(\boldsymbol{Q}, \omega) & =\frac{1}{2 \pi} \int_{-\infty}^{\infty} F(\boldsymbol{Q}, \infty) \exp (-i \omega t) d t+\frac{1}{2 \pi} \int_{-\infty}^{\infty} F_{1}(\boldsymbol{Q}, t) \exp (-i \omega t) d t \\
& =F(\boldsymbol{Q}, \infty) \cdot \delta(\omega)+\frac{1}{2 \pi} \int_{-\infty}^{\infty} F_{1}(\boldsymbol{Q}, t) \exp (-i \omega t) d t
\end{aligned}
$$

La forme et la largeur de la seconde composante dépendent de la nature et des temps caractéristiques des mouvements des noyaux. Dans le cas très simple où $F_{1}(\boldsymbol{Q}, t)$ décroît avec le temps de façon exponentielle, avec un seul temps caractéristique, $\tau$,

$$
\begin{gathered}
F(\boldsymbol{Q}, t)=[F(\boldsymbol{Q}, 0)-F(\boldsymbol{Q}, \infty)] \cdot \exp \left(-\frac{\boldsymbol{t}}{\tau}\right) \\
S(\boldsymbol{Q}, \omega)=F(\boldsymbol{Q}, \infty) . \delta(\omega)+\left[F(\boldsymbol{Q}, 0)-F(\boldsymbol{Q}, \infty] \cdot \frac{1}{\pi} \cdot \frac{\tau}{1+\omega^{2} \tau^{2}}\right.
\end{gathered}
$$

On aboutit à une composante quasiélastique de forme lorentzienne, sous-jacente à une composante purement élastique, de largeur nulle. En réalité la résolution d'un spectromètre est nécessairement limitée. C'est pourquoi le pic élastique n'est jamais infiniment étroit mais présente un profil (triangle, gaussienne ou lorentzienne) et une largeur caractéristiques de l'instrument. Si nous désignons par $R(\omega)$ cette fonction de résolution instrumentale (supposée normée à l'unité), la forme exacte des spectres expérimentaux sera donnée par le produit de convolution

$$
I(Q, \omega)=A_{\dot{e} c h} \int_{-\infty}^{+\infty} S\left(Q, \omega^{\prime}-\omega\right) R\left(\omega^{\prime}\right) d \omega^{\prime}
$$

$A_{\text {éch }}$ est une constante de mise à l'échelle. Supposons pour simplifier les calculs que la résolution de l'instrument soit une lorentzienne, de largeur $1 / \tau_{R}$.

$$
R(\omega)=\frac{1}{\pi} \cdot \frac{\tau_{R}}{1+\omega^{2} \tau_{R}^{2}}
$$

L'intensité neutronique observée expérimentalement sera 


$$
\begin{aligned}
I(Q, \omega) & =A_{\dot{e} c h}\left\{F(Q, \infty) \frac{1}{\pi} \cdot \frac{\tau_{R}}{1+\omega^{2} \tau_{R}^{2}}\right\}+A_{\dot{e} c h}\left\{[F(Q, 0)-F(Q, \infty)] \frac{1}{\pi} \cdot \frac{\tau_{\exp }}{1+\omega^{2} \tau_{\exp }{ }^{2}}\right\} \\
& =I_{\text {elas }}(Q, \omega)+I_{\text {quasi }}(Q, \omega)
\end{aligned}
$$

avec la largeur quasiélastique expérimentale

$$
\frac{1}{\tau_{\exp }}=\frac{1}{\tau_{R}}+\frac{1}{\tau}
$$

Alors que la largeur de la composante quasiélastique $I_{\text {quasi }}(Q, \omega)$ est directement liée aux temps caractéristiques des mouvements dont sont animés les atomes diffuseurs, l'importance de la contribution élastique $I_{\text {elas }}(Q, \omega)$ est donnée par la transformée de Fourier spatiale de leur distribution d'équilibre. Elle a la dimension d'un facteur de structure et traditionnellement elle est appelée "Facteur de structure élastique incohérent" et désignée par l'abréviation EISF (Elastic Incoherent Structure Factor).

Une caractéristique générale des échantillons offrant un désordre de translation est la possibilité pour l'atome diffuseur d'accéder à toutes les positions de l'espace. Ceci se traduit pour la fonction de corrélation de van Hove par

$$
\lim _{t \rightarrow \infty} G_{s}(\boldsymbol{r}, t)=G_{s}(\boldsymbol{r}, \infty)=0
$$

de sorte que pour la fonction de diffusion intermédiaire

$$
\lim _{\substack{T \rightarrow \infty \\ F_{T}}}(\boldsymbol{Q}, t)=F_{T}(\boldsymbol{Q}, \infty)=0
$$

Dans ces conditions on observera pour le facteur de structure dynamique l'absence d'un pic élastique. Au contraire, l'existence dans les spectres expérimentaux d'une part d'intensité diffusée élastique est le signe de la présence dans l'échantillon de diffuseurs dont le mouvement est localisé dans l'espace.

Telles que nous les avons définies, les fonctions de diffusion sont normées. En particulier, quand elle est intégrée sur tous les échanges d'énergie, la fonction de diffusion de rotation conduit à une valeur unité; en effet:

$$
\begin{aligned}
\int_{-\infty}^{+\infty} S_{R}(\boldsymbol{Q}, \omega) d \omega & =\int_{-\infty}^{+\infty}\left[\int_{-\infty}^{+\infty} F_{R}(\boldsymbol{Q}, t) \exp (-i \omega t) d t\right] d \omega \\
& =\int_{-\infty}^{+\infty} F_{R}(\boldsymbol{Q}, t) \delta(t) d t=F_{R}(\boldsymbol{Q}, 0)=1
\end{aligned}
$$

Ainsi l'EISF $F_{R}(Q, \infty)$ correspond à la part relative de l'intensité diffusée contenue dans le pic élastique. La conséquence directe et importante est que si l'on peut les spectres expérimentaux en leurs deux contributions, à savoir l'intensité diffusée sans changement d'énergie et celle diffusée de façon quasiélastique, il est possible, à partir des intensités intégrées correspondantes, d'évaluer pour chaque valeur de $Q$ le rapport 


$$
A_{0}(Q)=\frac{I_{\text {élas }}(Q)}{I_{\text {élas }}(Q)+I_{\text {quasi }}(Q)}
$$

qui est une détermination expérimentale de l'EISF.

La largeur du pic élastique $1 / \tau_{R}$ définit l'échelle de temps, $\tau_{R}$, sur laquelle les mouvements sont observables, par exemple $10^{-10} \mathrm{~s}$ pour un spectromètre à rétrodiffusion à haute résolution, $10^{-11}-10^{-12} \mathrm{~s}$ pour un spectromètre à temps de vol. Pour un spectromètre donné, des mouvements trop lents conduisent à des élargissements trop faibles pour être observés. Inversement des mouvements trop rapides produisent des élargissements considérables et la diffusion quasiélastique prend l'allure d'un bruit de fond dans le pied des spectres. Il faut absolument avoir toujours à l'esprit l'échelle de temps accessible au spectromètre que l'on utilise avant de tirer des conclusions sur la nature des mouvements. Pour illustrer ce point nous prendrons le cas de liquides moléculaires. L'existence de mouvements de déplacements à longue distance des molécules est une caractéristique fondamentale de l'état liquide. Pourtant l'analyse des spectres obtenus avec des spectromètres à temps de vol révèle souvent une contribution purement élastique, parce que la translation des molécules est trop lente pour être observée sur la gamme de temps accessible à ces instruments. L'analyse de la partie quasiélastique permet d'accéder aux temps caractéristiques associés à la rotation des molécules autour de leur centre de masse qui semble immobile. $\mathrm{Au}$ contraire quand ces échantillons sont étudiés à la même température au moyen de spectromètres à rétrodiffusion, leurs spectres ne montrent aucune composante purement élastique. Cette fois c'est la translation des molécules qui est à l'origine de l'élargissement observé. Quand à la rotation, elle est beaucoup trop large pour la gamme en énergie expérimentale et elle se manifeste sous forme d'un bruit de fond plat.

\section{LES SPECTROMETRES POUR L'ANALYSE DES MOUVEMENTS DIFFUSIFS}

Au contraire des spectromètres trois axes les spectromètres à temps de vol et à rétro diffusion sont conçus pour enregistrer simultanément les neutrons diffusés dans une grande gamme de vecteurs d'onde et de transferts d'énergie. Ils sont utilisés pour mesurer $S(Q, \omega)$ pour des liquides ou des échantillons désordonnés ou encore des spectres de diffusion magnétique qui varient peu avec $\boldsymbol{Q}$. On trouvera une revue détaillée de ces instruments dans [12].

La spectroscopie neutronique par temps de vol est une méthode puissante pour étudier sur une large gamme dynamique les processus dynamiques dans les solides polycristallins et les solides désordonnés. Le flux continu de neutrons est découpé en courtes bouffées afin de déterminer l'instant origine du parcours des neutrons. Plusieurs centaines de détecteurs sont placés de façon à récolter simultanément les neutrons diffusés pour plusieurs valeurs du vecteur d'onde. Ils sont situés non seulement dans le plan de diffusion mais aussi au dessus et au dessous de celui-ci. Le premier instrument de ce type fut IN5 à l'ILL (Grenoble, France [13]), qui utilise plusieurs disques ("choppers") séparés afin de créer des bouffées de neutrons d'une énergie bien caractéristique. Par la suite d'autres instruments de ce type on été construits : MIBEMOL au LLB (Saclay, France [14]), NEAT au HMI (Berlin, Allemagne [15]) et DCS au NIST (Gaithersburg, USA [16]), avec un certain nombre d'améliorations. D'autres spectromètres à temps de vol utilisent un cristal monochromateur associé à un chopper de Fermi. De nombreux instruments de ce type existent pour les neutrons thermiques. Pour les neutrons froids, l'utilisation de techniques de focalisation temporelle a permis un gain substantiel en intensité (IN6 à l'ILL, FOCUS au PSI (Villigen, Suisse [17]), FCS au NIST.

Avec les sources à spallation on utilise souvent des spectromètres temps de vol inversés. IRIS au RAL (Royaume-Uni [18]) est le premier spectromètre construit sur ce principe. Des bouffés de neutrons polychromatiques viennent frapper l'échantillon. Leur fréquence de répétition des bouffées est directement liée à la période de la source elle-même. Après le processus de diffusion, des cristaux analyseurs renvoient des neutrons ayant une énergie bien déterminée dans des détecteurs où ils sont comptés dans différents canaux en temps de vol. L'avantage principal de IRIS est d'être très souple 
d'utilisation avec une large gamme dynamique. Son succès a inspiré beaucoup d'instruments similaires sur d'autres sources à spallation.

Les neutrons diffusés inélastiquement par une cible gagnent ou perdent de l'énergie et donc subissent une variation de vitesse. Il est clair que la partie du spectre correspondant à une perte d'énergie des neutrons est limitée par l'intervalle de temps entre deux bouffées. L'énergie d'un spectromètre à temps de vol est déterminée par le temps de parcours des neutrons et l'incertitude sur l'énergie qui est proportionnelle à la durée de la bouffée. On obtient les mesures les plus précises avec des machines ayant de grandes dimensions (plusieurs mètres) et des disques tournant à très grande vitesse.

$\mathrm{Si}$ on tient compte de la valeur de l'énergie incidente des neutrons, il est clair que pour un angle de diffusion donné entre $\boldsymbol{k}_{\boldsymbol{i}}$ et $\boldsymbol{k}_{\boldsymbol{f}}$, le vecteur de diffusion, $\boldsymbol{Q}$, ne peut pas rester constant si le transfert d'énergie varie. Ce point doit être pris en compte dans le traitement des données.

Les spectromètres à rétrodiffusion sont des versions particulières de spectromètres trois axes conçues pour travailler avec des angles de diffraction de Bragg sur le monochromateur et sur les analyseurs égaux ou très proches de $90^{\circ}$. En différentiant la loi de Bragg on obtient

$$
\frac{\Delta \lambda}{\lambda}=\frac{\Delta d}{d}+\frac{\Delta \theta}{\tan \theta}
$$

La meilleure résolution s'obtient pour $\theta=90^{\circ}$ quand seule la dispersion des distances réticulaire contribue. On obtient des résolutions de $1 \mu \mathrm{eV}$ avec des incertitude relatives $\Delta d / d$ de l'ordre de $10^{-5}$. La spectroscopie en rétrodiffusion est une technique importante pour avoir accès aux temps caractéristiques longs. L'analyse se fait à énergie finale constante, en faisant varier l'énergie incidente soit par effet Doppler (IN10, IN16 à l'ILL) ou par chauffage du cristal monochromateur (IN13 à l'ILL). Les spectromètres à rétrodiffusion peuvent travailler sur une grande gamme de transfert de moment et permettent d'accéder à des temps compris entre 0,01 ps et 100 ps. Leur résolution est déterminée principalement par la divergence du faisceau de neutrons.

La spectroscopie neutronique par écho de spin est quelque peu différente de celles par temps de vol et par rétrodiffusion. Le principe en a été introduit par Mezei en 1972 [22, 23]. Tandis que les spectromètres à rétrodiffusion ou par temps de vol mesurent dans l'espace des fréquences, et déterminent $\mathrm{S}(\boldsymbol{Q}, \omega)$, la spectrométrie par écho de spin mesure dans l'espace des temps et détermine directement la fonction de diffusion intermédiaire $S(\boldsymbol{Q}, t)$. En plus de l'instrument originel IN11, deux autres spectromètres ont été construits par la suite MESS au LLB et IN15 à l'ILL.

The principle was introduced by F. Mezei in $1972[19,20]$. While BS and TOF spectrometers measures in frequency space, and thus determines $S(\boldsymbol{Q}, \omega)$, neutron spin echo spectroscopy measure in time-space and directly determine the intermediate scattering function $S(\boldsymbol{Q}, t)$. Besides the original instrument $\mathrm{IN} 11$ two other spectrometers were later built: MESS at LLB and IN15 at ILL. En 1987 Gähler et Golub ont suggéré un nouveau type de spectromètre désigné sous le nom de "spectromètre à écho de spin par résonance" ou encore " spectromètre à écho de spin en champ nul" qui utilise des bobines radiofréquence directement inspirés de la RMN [21]. Des instruments de ce type ont été construits au HMi, au LLB ou sont en cours de développement (Münich).

\section{DIFFUSION A LONGUE DISTANCE}

\subsection{La loi de Fick}

Les mouvements de diffusion à longue distance sont associés aux phénomènes de transport. Ils interviennent dans tous les états de la matière, qu'il s'agisse de gaz, de liquides ou de solides. Leur compréhension est d'une importance primordiale dans le domaine de la recherche appliquée: polymères, diffusion de l'hydrogène dans les métaux, déplacement d'ions dans les électrolytes solides, déplacement 


\section{NEUTRONS ET SYSTÈMES DÉSORDONNÉS}

d'espèces chimiques dans les matériaux poreux, catalyse, etc...Parmi les techniques expérimentales utilisées pour caractériser ces phénomènes, la diffusion incohérente des neutrons fournit des informations sur les aspects spatiaux et temporaux des mécanismes élémentaires mis en jeu [22, 23].

Supposons que le seul mouvement pertinent de l'atome diffuseur soit un mouvement de translation, purement aléatoire. Cette situation correspond au mouvement brownien d'un ensemble de particules en très faible interaction, découvert par Brown en 1827 lors de l'observation de petites particules en suspension dans un liquide et étudié indépendamment par Einstein, Smoluchowski et Langevin. L'équation phénoménologique de base décrivant l'évolution spatiale et temporelle de la concentration des atomes diffuseurs, $c(r, t)$ est la seconde loi de Fick dont les travaux remontent à 1855, et qui s'écrit:

$$
\frac{\partial c(\boldsymbol{r}, t)}{\partial t}=D \nabla^{2} c(\boldsymbol{r}, t)=D\left[\frac{\partial^{2} c(\boldsymbol{r}, t)}{\partial x^{2}}+\frac{\partial^{2} c(\boldsymbol{r}, t)}{\partial y^{2}}+\frac{\partial^{2} c(\boldsymbol{r}, t)}{\partial z^{2}}\right]
$$

Cette équation définit le coefficient de diffusion, $D$, à un niveau macroscopique. La diffusion neutronique étudie les phénomènes à l'échelle microscopique. Le système étant supposé à l'équilibre, la fonction d'autocorrélation de van Hove, $G_{s}(r, t)$, c'est-à-dire la probabilité pour un atome, initialement situé à l'origine, d'être en $\boldsymbol{r}$ à l'instant $t$, se relie simplement à la concentration atomique par unité de volume:

$$
G_{s}(\boldsymbol{r}, t)=\frac{c(\boldsymbol{r}, t)}{N}
$$

où $N$ est le nombre total d'atomes. Dès lors, $G_{s}(r, t)$ satisfait à l'équation de Fick dont une solution est une gaussienne normalisée

$$
G_{s}(r, t)=\frac{1}{(4 \pi D t)^{3 / 2}} \exp \left(-\frac{r^{2}}{4 D t}\right)
$$

Cette expression remplit la condition initiale $G_{s}(r, t)=\delta(r)$. L'absence de force d'entraînement dans une direction quelconque conduit à une fonction isotrope dans l'espace.

Le déplacement carré moyen d'un atome est proportionnel au temps

$$
\left\langle r^{2}(t)\right\rangle=6 D t
$$

Il correspond à la loi de diffusion libre d'Einstein (marche au hasard) [24].

En prenant la transformée de Fourier spatiale de l'équation de la seconde loi de Fick, on obtient la fonction de diffusion intermédiaire, qui est aussi une gaussienne isotrope mais en $Q$ cette fois

$$
F(Q, i)=F(Q, t)=\exp \left(-D Q^{2} t\right)
$$

Il est intéressant de remarquer que chacun des atomes pouvant s'éloigner de l'origine d'une distance arbitraire,

$$
\lim _{t \rightarrow \infty} F(Q, t)=0
$$

De sorte que l'EISF est nul. La transformée de Fourier temporelle conduit au facteur de structure dynamique. Il est isotrope en $Q$ et son profil est celui d'une lorentzienne

$$
S_{i n c}(Q, \omega)=\frac{1}{\pi} \frac{D Q^{2}}{\omega^{2}+\left(D Q^{2}\right)^{2}}
$$


dont la largeur à mi-hauteur, $\Gamma$, croît en fonction du transfert de moment selon une loi parabolique.

Ainsi en portant la largeur des spectres obtenus pour différentes valeurs de $Q^{2}$, on doit obtenir une droite, dont la pente fournit directement la constante de diffusion. C'est ce que l'on observe effectivement avec le paraxylène liquide (figure 10) Toutefois, cette loi n'est véritablement vérifiée qu'aux faibles valeurs du transfert de moment, correspondant aux grandes distances (plusieurs dizaines de diamètres moléculaires). Aux plus grandes valeurs de $Q$, la courbe obtenue s'écarte systématiquement de la droite théorique car au niveau des petites distances d'observation, le milieu n'apparaît plus comme continu et les mécanismes microscopiques sous-jacents à la diffusion (sauts) entrent en jeu.

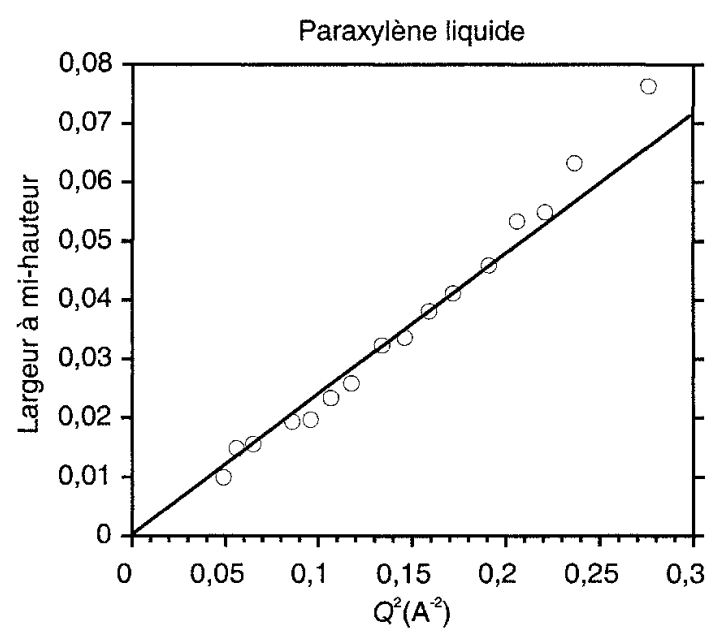

Figure 10 : Evolution de largeur à mi-hauteur des spectres quasiélastiques obtenus avec le paraxylène liquide, en fonction du transfert de moment, $Q$. La droite correspond à la loi de Fick qui est vérifiée aux faibles valeurs de $Q$. Mais on observe un écart systématique aux plus grandes valeurs, quand l'échelle des distances d'observation devient petite et que les mécanismes élémentaires du processus de diffusion apparaissent.

\subsection{Diffusion par sauts}

Comme nous l'avons indiqué, l'écart à la loi théorique de Fick traduit le caractère discontinu de la diffusion. Différents modèles de diffusion par sauts ont été imaginés, pour s'appliquer à différents systèmes: diffusion de l'hydrogène dans les métaux, molécules adsorbées dans des milieux poreux (zéolithes) ou liquides moléculaires (eau).

Chudley et Elliott [25] ont été parmi les premiers à développer l'un de ces modèles, pour un liquide dans lequel il subsiste un ordre à courte distance. Les hypothèses sont les suivantes: pendant un intervalle de temps $\tau$ l'atome diffuseur reste au voisinage d'un site particulier, effectuant des vibrations autour d'une position d'équilibre moyenne. Puis, il se déplace vers un autre site, dans un laps de temps suffisamment court pour que ce changement de position d'équilibre puisse être assimilé à un saut instantané. La distance de ce saut, $l$, est supposée beaucoup plus grande que les déplacements de vibration autour des différentes positions d'équilibre.

Ecrit initialement pour des liquides, le modèle de Chudley et Elliott a trouvé de nombreuses applications dans différents domaines. Il s'applique bien à l'étude de la diffusion de l'hydrogène atomique adsorbé dans les métaux [23]. Dans ce cas les sites d'équilibre sont répartis sur un réseau de Bravais. En se limitant aux sauts vers les sites les plus proches, l'équation de diffusion analogue à l'équation de Fick (équation maîtresse) est 


$$
\frac{\partial P(\boldsymbol{r}, t)}{\partial t}=\frac{1}{n \tau} \sum_{i=1}^{n}\left[P\left(\boldsymbol{r}+\boldsymbol{l}_{i}, t\right)-P(\boldsymbol{r}, t)\right]
$$

dans laquelle $P(r, t)$ désigne la probabilité pour l'atome d'occuper le site en $r$ et où la somme porte sur les $n$ sites plus proches voisins situés aux distances $l_{i}$. La transformation de Fourier de cette expression conduit pour la fonction de diffusion intermédiaire $F(\boldsymbol{Q}, t)$ à une équation différentielle

$$
\frac{\partial F(\boldsymbol{Q}, t)}{\partial t}=\left[\frac{1}{n \tau} \sum_{i=1}^{n}\left\{1-\exp \left(-i \boldsymbol{Q} \boldsymbol{l}_{t}\right)\right\}\right] F(\boldsymbol{Q}, t)
$$

qui admet la solution

$$
F(\boldsymbol{Q}, t)=\exp \left(-\frac{1}{n \tau} \sum_{i=1}^{n}\left\{1-\exp \left(-i \boldsymbol{Q} \cdot \boldsymbol{l}_{i}\right)\right\} t\right)
$$

La fonction de diffusion intermédiaire n'est plus isotrope et dépend de l'orientation du vecteur $\boldsymbol{Q}$ par rapport aux directions cristallographiques. Il en est de même du facteur de structure dynamique

$$
S_{i n c}(\boldsymbol{Q}, \omega)=\frac{1}{\pi} \frac{\Delta \omega(\boldsymbol{Q})}{\omega^{2}+(\Delta \omega(\boldsymbol{Q}))^{2}}
$$

dont la demi-largeur à mi-hauteur

$$
\Delta \omega(\boldsymbol{Q})=\frac{1}{n \tau} \sum_{i=1}^{n}\left\{1-\exp \left(-i \boldsymbol{Q} \boldsymbol{I}_{\boldsymbol{i}}\right)\right\}
$$

varie avec la direction d'observation des neutrons diffusés. Dans le cas de sauts vers des sites de type différents faisant intervenir plusieurs probabilité de saut, l'expression de la demi-largeur à mi-hauteur est sensiblement plus compliquée. On peut mettre à profit sa dépendance avec la direction de $\boldsymbol{Q}$ pour discriminer entre plusieurs hypothèses de modèles dynamiques. Dans le cas de poudres l'expression de la loi de diffusion doit être moyennée pour tenir compte de toutes les orientations possibles des cristallites par rapport au vecteur de diffusion. Il n'existe pas d'expression analytique et cette moyenne est à effectuer numériquement. On aboutit à une somme de fonctions lorentziennes de largeur différentes. Aux petites valeurs de $Q$ le profil de $S_{\text {inc }}(Q, \omega)$ ressemble à celui d'une lorentzienne mais des différences très nettes apparaissent aux plus grandes valeurs de $Q$.

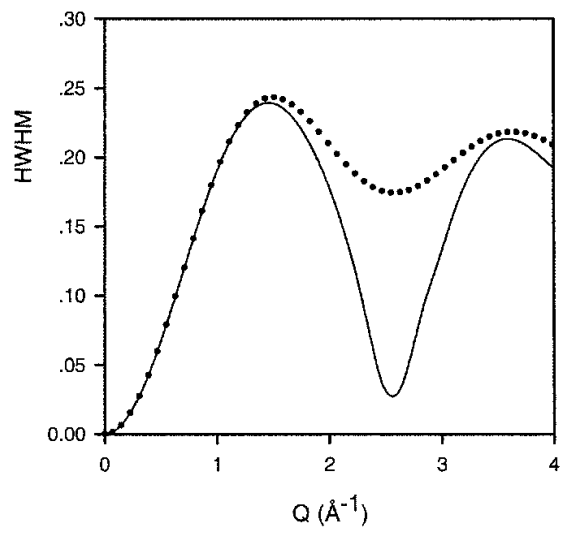

Figure 11: Elargissement des spectres quasiélastiques avec le transfert de moment, $Q$, selon le modèle de Chudley-Eliott. Les calculs ont été faits pour des sauts entre les sites octaédriques équivalents d'un réseau de Bravais. La distance de saut a été prise égale à $3 \AA$. La courbe pointillée correspond à la moyenne isotrope des largeurs selon la relation (97). La courbe en trait continu est la largeur obtenue après avoir fait une moyenne du signal (d'après [8]). 
La description d'un liquide de Chudley et Eliott est celle de molécules, enfermées dans une cage formée des autres molécules, s'échappant de temps à autre vers une autre cage. La distance de saut $l$ est identique pour tous les sites, mais la direction des sauts est aléatoire, ce qui conduit ces auteurs à prendre la moyenne spatiale de la demi-largeur à mi-hauteur

$$
\begin{aligned}
\Delta \omega(Q) & =\frac{1}{\tau}\left\{1-\frac{1}{2} \int_{0}^{\pi} \exp (-i Q l \cos \theta) \sin \theta d \theta\right\} \\
& \left.=\frac{1}{\tau}\left\{1-\frac{\sin (Q l)}{Q l}\right)\right\}
\end{aligned}
$$

Cette expression diffère sensiblement du résultat correct obtenu en prenant la véritable demi-largeur à mihauteur du profil du spectre obtenu en moyennant les fonctions lorentziennes comme le montre la figure 11 .

Néanmoins, aux faibles valeurs de $Q$ les deux courbes cö̈ncident. En prenant le développement limité de la fonction sinus, on trouve

$$
\begin{aligned}
\lim _{Q \rightarrow 0} \Delta \omega(Q) & =\frac{1}{\tau}\left\{1-1+\frac{Q^{2} l^{2}}{3 !}+\ldots\right\} \\
& =\frac{Q^{2} l^{2}}{6 \tau}
\end{aligned}
$$

Ainsi, aux faibles valeurs de $Q$, le modèle de Chudley-Elliott redonne la loi macroscopique en $D Q^{2}$, la constante de diffusion, $D$, étant reliée au temps de résidence $\tau$, par la relation d'Einstein

$$
D=\frac{l^{2}}{6 \tau}
$$

Par contre, aux grandes valeurs de $Q$, le terme oscillatoire dans les équations (96) et (97) devient négligeable et la demi-largeur à mi-hauteur tend vers une valeur constante $1 / \tau$ égale à la probabilité de saut.

Le modèle de Chudley-Elliott se généralise au cas d'une distribution de distances de sauts, $g(l)$

$$
\left.\Delta \omega(Q)=\frac{1}{\tau}\left\{1-\int \frac{\sin (Q l)}{Q l} g(l) d l\right)\right\}
$$

En particulier, Hall et Ross [26] en utilisant une distribution de la forme

$$
g(l)=\frac{2 l^{2}}{\sqrt{2 \pi} l_{0}^{3}} \exp \left(-\frac{l^{2}}{2 l_{0}^{2}}\right)
$$

aboutissent à l'expression suivante de la demi-largeur:

$$
\Delta \omega(Q)=\frac{1}{\tau}\left\{1-\exp \left(-\frac{Q^{2} l_{0}^{2}}{2}\right)\right\}
$$

D'autres modèles de diffusion translationnelle ont été imaginés. Citons en particulier celui de Singwi et Sjölander [27] qui suppose pour les atomes une succession d'états vibratoires et diffusifs. Ce modèle a été appliqué avec succès à la description de la diffusion des molécules dans l'eau liquide. La relation entre les deux modèles a été mise en évidence par Egelstaff [22] qui a montré que l'utilisation d'une distribution de la forme

$$
g(l)=l \exp \left(-\frac{l}{l_{0}}\right)
$$

dans le modèle de Chudley-Elliott conduisait à une version simplifiée de celui de Singwi et Sjölander avec un temps de saut suffisamment court par rapport au temps de résidence pour pouvoir être négligé. Citons enfin Jobic [28] qui a développé un modèle tenant compte de la délocalisation des molécules sur leurs sites. Suivant l'importance plus ou moins grande de cette délocalisation, il retrouve les deux limites correspondant aux modèles de Chudley-Elliott ou Hall et Ross. 
Les demi-largeurs des profils de diffusion de tous ces modèles montrent un comportement en $D Q^{2}$ correspondant à la loi de Fick aux faibles valeurs de $Q$. Parmi eux, seul le modèle de Chudley et Elliott présente un comportement oscillatoire, toutes les largeurs tendant asymptotiquement vers $1 / \tau$ aux grandes valeurs de $Q$.

\section{MOUVEMENTS LOCALISES}

L'écrasante majorité des solides est formée de composés formés de deux ou plusieurs éléments. Dans beaucoup de ces systèmes, l'espèce diffusante (atome ou molécule) occupe des sites cristallins distincts, pouvant correspondre à des valeurs différentes de l'énergie. Si des groupes de sites voisins sont séparés des autres par des distances plus importantes ou par des barrières d'énergie plus importantes, les déplacements d'atomes piégés dans ces sites peuvent se résumer à des passages d'un site du groupe à l'autre: leur diffusion est limitée dans l'espace. Un exemple de situation analogue est celui d'une molécule dans un réseau cristallin ou sur un site d'adsorption, qui se réoriente entre plusieurs positions d'équilibre. Alors les atomes de cette molécule ne sont autorisés à effectuer qu'un nombre bien déterminé de déplacements bien précis. Il est clair que dans chacun des cas, sous l'effet des fluctuations thermiques, des déplacements occasionnels pourront se produire vers d'autres groupes de sites atomiques ou vers d'autres sites moléculaires d'adsorption. De même par l'intermédiaire des lacunes existant dans un cristal moléculaire une molécule pourra migrer vers un autre site du réseau. Mais si le temps caractéristique associé à ces déplacements de diffusion est suffisamment long les deux types de mouvements pourront être considérés comme dynamiquement indépendants et la fonction de diffusion dynamique sera le produit de convolution des deux fonctions de diffusion séparées.

\subsection{Sauts sur deux sites non équivalents}

Nous allons considérer le cas le plus simple d'une particule capable d'effectuer des sauts entre deux positions d'équilibre non équivalentes, repérées par $\boldsymbol{r}_{1}$ et $\boldsymbol{r}_{2}$. Soient $\tau_{1}$ et $\tau_{2}$ les temps moyens de résidence dans ces sites respectifs. Les probabilités $p\left(r_{1}, t\right)$ et $p\left(r_{2}, t\right)$ de trouver la particule au temps $t$ sur chacun des sites sont gouvernées par le système d'équations différentielles

$$
\begin{aligned}
& \frac{d p\left(\boldsymbol{r}_{1}, t\right)}{d t}=-\frac{1}{\tau_{1}} p\left(\boldsymbol{r}_{1}, t\right)+\frac{1}{\tau_{2}} p\left(\boldsymbol{r}_{2}, t\right) \\
& \frac{d p\left(\boldsymbol{r}_{2}, t\right)}{d t}=\frac{1}{\tau_{1}} p\left(\boldsymbol{r}_{1}, t\right)-\frac{1}{\tau_{2}} p\left(\boldsymbol{r}_{2}, t\right)
\end{aligned}
$$

la solution d'équilibre s'obtient en annulant les dérivées dans les équations précédentes

$$
\begin{aligned}
& p\left(r_{1}, 0\right)=p\left(r_{1}, \infty\right)=\frac{\tau_{1}}{\tau_{1}+\tau_{2}} \\
& p\left(r_{2}, 0\right)=p\left(r_{2}, \infty\right)=\frac{\tau_{2}}{\tau_{1}+\tau_{2}}
\end{aligned}
$$

Les solutions font intervenir les valeurs propres et les vecteurs propres du système. Elles s'expriment sous la forme

$$
\begin{aligned}
& p\left(\boldsymbol{r}_{1}, t\right)=A+B \exp (-t / \tau) \\
& p\left(\boldsymbol{r}_{2}, t\right)=\frac{\tau_{2}}{\tau_{1}} A-B \exp (-t / \tau)
\end{aligned}
$$

dans laquelle le temps caractéristique, $\tau$ est défini comme 


$$
\frac{1}{\tau}=\frac{1}{\tau_{1}}+\frac{1}{\tau_{2}}
$$

et où les constantes $A$ et $B$ sont à déterminer à partir des conditions initiales. En particulier si on fait l'hypothèse d'une particule située initialement sur le site 1 , on peut écrire

$$
\begin{aligned}
& p\left(\boldsymbol{r}_{1}, 0 ; \boldsymbol{r}_{1}, 0\right)=1=A+B \\
& p\left(\boldsymbol{r}_{2}, 0 ; \boldsymbol{r}_{1}, 0\right)=0=\frac{\tau_{2}}{\tau_{1}} A-B
\end{aligned}
$$

et on obtient les probabilités conditionnelles

$$
\begin{aligned}
& p\left(r_{1}, t ; r_{1}, 0\right)=\frac{\tau_{1}}{\tau_{1}+\tau_{2}}+\frac{\tau_{2}}{\tau_{1}+\tau_{2}} \exp (-t / \tau) \\
& p\left(r_{2}, t ; r_{1}, 0\right)=\frac{\tau_{2}}{\tau_{1}+\tau_{2}}-\frac{\tau_{2}}{\tau_{1}+\tau_{2}} \exp (-t / \tau)
\end{aligned}
$$

De manière analogue, en supposant la particule située sur le site 2 à l'instant $t=0$, on écrira

$$
\begin{aligned}
& p\left(\boldsymbol{r}_{1}, 0 ; \boldsymbol{r}_{2}, 0\right)=0=A+B \\
& p\left(\boldsymbol{r}_{2}, 0 ; \boldsymbol{r}_{1}, 0\right)=1=\frac{\tau_{2}}{\tau_{1}} A-B
\end{aligned}
$$

ce qui conduit aux probabilités conditionnelles

$$
\begin{aligned}
& p\left(r_{1}, t ; r_{2}, 0\right)=\frac{\tau_{1}}{\tau_{1}+\tau_{2}}-\frac{\tau_{1}}{\tau_{1}+\tau_{2}} \exp (-t / \tau) \\
& p\left(r_{2}, t ; r_{2}, 0\right)=\frac{\tau_{2}}{\tau_{1}+\tau_{2}}+\frac{\tau_{1}}{\tau_{1}+\tau_{2}} \exp (-t / \tau)
\end{aligned}
$$

Puisqu'à chaque instant la particule se situe de façon certaine sur l'un des deux sites, ces probabilités sont normées.

$$
\begin{aligned}
& p\left(\boldsymbol{r}_{1}, t ; \boldsymbol{r}_{1}, 0\right)+p\left(\boldsymbol{r}_{2}, t ; \boldsymbol{r}_{1}, 0\right)=1 \\
& p\left(\boldsymbol{r}_{1}, t ; \boldsymbol{r}_{2}, 0\right)+p\left(\boldsymbol{r}_{2}, t ; \boldsymbol{r}_{2}, 0\right)=1
\end{aligned}
$$

On vérifie aisément qu'au temps infini, ces solutions conduisent aux expressions d'équilibre:

$$
\begin{aligned}
& \lim _{i \rightarrow \infty} p\left(\boldsymbol{r}_{1}, t ; r_{2}, 0\right)=\lim _{t \rightarrow \infty} p\left(\boldsymbol{r}_{1}, t ; r_{1}, 0\right)=p\left(\boldsymbol{r}_{1}, \infty\right)=\frac{\tau_{1}}{\tau_{1}+\tau_{2}} \\
& \lim _{t \rightarrow \infty} p\left(\boldsymbol{r}_{2}, t ; r_{1}, 0\right)=\lim _{t \rightarrow \infty} p\left(\boldsymbol{r}_{2}, t ; r_{2}, 0\right)=p\left(\boldsymbol{r}_{2}, \infty\right)=\frac{\tau_{2}}{\tau_{1}+\tau_{2}}
\end{aligned}
$$

ce qui signifie que la particule a complètement oublié son histoire passée. En outre, à un instant quelconque, on peut développer la probabilité d'occupation de chacun des sites par la particule sous la forme 


$$
\begin{aligned}
& p\left(\boldsymbol{r}_{1}, t\right)=p\left(\boldsymbol{r}_{1}, t ; \boldsymbol{r}_{1}, 0\right) p\left(\boldsymbol{r}_{1}, 0\right)+p\left(\boldsymbol{r}_{1}, t ; \boldsymbol{r}_{2}, 0\right) p\left(\boldsymbol{r}_{2}, 0\right) \\
& p\left(\boldsymbol{r}_{2}, t\right)=p\left(\boldsymbol{r}_{2}, t ; \boldsymbol{r}_{1}, 0\right) p\left(\boldsymbol{r}_{1}, 0\right)+p\left(\boldsymbol{r}_{2}, t ; \boldsymbol{r}_{2}, 0\right) p\left(\boldsymbol{r}_{2}, 0\right)
\end{aligned}
$$

En explicitant ces expressions on obtient

$$
\begin{aligned}
& p\left(\boldsymbol{r}_{1}, t\right)=\frac{\tau_{1}}{\tau_{1}+\tau_{2}}\left[\frac{\tau_{1}}{\tau_{1}+\tau_{2}}+\frac{\tau_{2}}{\tau_{1}+\tau_{2}} \exp (-t / \tau)\right]+\frac{\tau_{2}}{\tau_{1}+\tau_{2}}\left[\frac{\tau_{1}}{\tau_{1}+\tau_{2}}-\frac{\tau_{1}}{\tau_{1}+\tau_{2}} \exp (-t / \tau)\right] \\
& p\left(\boldsymbol{r}_{2}, t\right)=\frac{\tau_{1}}{\tau_{1}+\tau_{2}}\left[\frac{\tau_{2}}{\tau_{1}+\tau_{2}}-\frac{\tau_{2}}{\tau_{1}+\tau_{2}} \exp (-t / \tau)\right]+\frac{\tau_{2}}{\tau_{1}+\tau_{2}}\left[\frac{\tau_{2}}{\tau_{1}+\tau_{2}}+\frac{\tau_{1}}{\tau_{1}+\tau_{2}} \exp (-t / \tau)\right]
\end{aligned}
$$

quí conduisent à

$$
\begin{aligned}
p\left(\boldsymbol{r}_{1}, t\right) & =\frac{\tau_{1}}{\tau_{1}+\tau_{2}} \\
p\left(\boldsymbol{r}_{2}, t\right) & =\frac{\tau_{2}}{\tau_{1}+\tau_{2}} \\
p\left(\boldsymbol{r}_{1}, t\right)+p\left(\boldsymbol{r}_{2}, t\right) & =1
\end{aligned}
$$

Ainsi, à tout instant le système est en équilibre. Cette remarque est importante car dans le cas de simulations de dynamique moléculaires, elle autorise de calculer la fonction de diffusion intermédiaire à partir de la trajectoire simulée d'une particule au cours du temps en remplaçant la moyenne thermique par une moyenne sur des positions prises au cours du temps.

Dans le cadre d'un calcul analytique, la fonction de diffusion intermédiaire s'évalue à partir des probabilités conditionnelles, en tenant compte de la probabilité de chacune des configurations initiales

$$
\begin{aligned}
F(\boldsymbol{Q}, t) & =\left[p\left(\boldsymbol{r}_{1}, t ; \boldsymbol{r}_{1}, 0\right)+p\left(\boldsymbol{r}_{2}, t ; \boldsymbol{r}_{1}, 0\right) \exp \left\{i Q .\left(\boldsymbol{r}_{2}-\boldsymbol{r}_{1}\right)\right\}\right] p\left(\boldsymbol{r}_{1}, 0\right) \\
& +\left[p\left(\boldsymbol{r}_{1}, t ; \boldsymbol{r}_{2}, 0\right) \exp \left\{i Q .\left(\boldsymbol{r}_{1}-\boldsymbol{r}_{2}\right)\right\}+p\left(\boldsymbol{r}_{2}, t ; \boldsymbol{r}_{2}, 0\right)\right] p\left(\boldsymbol{r}_{2}, 0\right)
\end{aligned}
$$

Sous forme explicite

$$
\begin{aligned}
F(\boldsymbol{Q}, t) & =\left[\frac{\tau_{1}}{\tau_{1}+\tau_{2}}+\frac{\tau_{2}}{\tau_{1}+\tau_{2}} \exp (-t / \tau)+\left(\frac{\tau_{2}}{\tau_{1}+\tau_{2}}-\frac{\tau_{2}}{\tau_{1}+\tau_{2}} \exp (-t / \tau)\right) \exp \left\{i \boldsymbol{Q} .\left(\boldsymbol{r}_{2}-\boldsymbol{r}_{1}\right)\right\}\right] \frac{\tau_{1}}{\tau_{1}+\tau_{2}} \\
& +\left[\left(\frac{\tau_{1}}{\tau_{1}+\tau_{2}}-\frac{\tau_{1}}{\tau_{1}+\tau_{2}} \exp (-t / \tau)\right) \exp \left\{i \boldsymbol{Q} \cdot\left(\boldsymbol{r}_{1}-\boldsymbol{r}_{2}\right)\right\}+\frac{\tau_{2}}{\tau_{1}+\tau_{2}}+\frac{\tau_{1}}{\tau_{1}+\tau_{2}} \exp (-t / \tau)\right] \frac{\tau_{2}}{\tau_{1}+\tau_{2}}
\end{aligned}
$$

En définissant un vecteur de saut $\boldsymbol{r}=\boldsymbol{r}_{1}-\boldsymbol{r}_{2}$ on aboutit à

$$
F(\boldsymbol{Q}, t)=\frac{1}{\left(\tau_{1}+\tau_{2}\right)^{2}}\left[\tau_{1}^{2}+\tau_{2}^{2}+2 \tau_{1} \tau_{2} \cos (\boldsymbol{Q} \cdot \boldsymbol{r})\right]+\frac{2 \tau_{1} \tau_{2}}{\left(\tau_{1}+\tau_{2}\right)^{2}}[1-\cos (\boldsymbol{Q} \cdot \boldsymbol{r})] \exp (-t / \tau)
$$

L'expression précédente fait intervenir l'orientation du vecteur de diffusion $\boldsymbol{Q}=Q(\theta, \varphi)$ par rapport au vecteur de diffusion. Dans le cas d'un échantillon polycristallin elle est à moyenner sur toutes leurs orientations respectives possibles. 


$$
F(Q, t)=\iint F(Q, t) \sin \theta d \theta d \varphi
$$

Contrairement au cas de la diffusion à longue distance sur un réseau de sites d'adsorption, l'argument de l'exponentielle ne dépend pas du vecteur de diffusion. C'est pourquoi la moyenne de poudre aboutit cette fois à une expression analytique, faisant intervenir une exponentielle unique

$$
F(Q, t)=\frac{1}{\left(\tau_{1}+\tau_{2}\right)^{2}}\left[\tau_{1}^{2}+\tau_{2}^{2}+2 \tau_{1} \tau_{2} \frac{\sin (Q \cdot r)}{Q \cdot r}\right]+\frac{2 \tau_{1} \tau_{2}}{\left(\tau_{1}+\tau_{2}\right)^{2}}\left[1-\frac{\sin (Q \cdot r)}{Q \cdot r}\right] \exp (-t / \tau)
$$

Le terme indépendant du temps qui apparât dans cette expression traduit la localisation de la particule dans l'espace, de sorte que au temps infini, le facteur de structure élastique incohérent (EISF) vaut

$$
\lim _{t \rightarrow \infty} F(Q, t)=F(Q, \infty)=\frac{1}{\left(\tau_{1}+\tau_{2}\right)^{2}}\left[\tau_{1}^{2}+\tau_{2}^{2}+2 \tau_{1} \tau_{2} \frac{\sin (Q . r)}{Q \cdot r}\right]
$$

Ce terme est représenté sur la figure 12 , pour différentes valeurs du rapport $\tau_{2} / \tau_{1}$. Il est de nature oscillatoire, de pseudo-période $Q . r$ et admet pour limite aux grands transferts de moments

$$
\lim _{Q \rightarrow \infty} F(Q, \infty)=\frac{\tau_{1}^{2}+\tau_{2}^{2}}{\left(\tau_{1}+\tau_{2}\right)^{2}}
$$

La courbe la plus basse de l'EISF est obtenue lorsque $\tau_{2}=\tau_{1}$, ce qui correspond à deux sites équivalents énergétiquement. Dans ces conditions la particule se répartit également entre les deux positions:

$$
\begin{aligned}
& F_{\min }(Q, t)=\frac{1}{2}\left[1+\frac{\sin (Q \cdot r)}{Q \cdot r}\right]+\frac{1}{2}\left[1-\frac{\sin (Q \cdot r)}{Q \cdot r}\right] \exp (-t / \tau) \\
& \lim _{t \rightarrow \infty} F_{\min }(Q, t)=F_{\min }(Q, \infty)=\frac{1}{2}\left[1+\frac{\sin (Q \cdot r)}{Q \cdot r}\right] \\
& \lim _{Q \rightarrow \infty} F_{\min }(Q, \infty)=\frac{1}{2}
\end{aligned}
$$

Inversement lorsque la particule reste indéfiniment sur l'un des sites quel qu'il soit

$$
\lim _{\tau_{1} \rightarrow \infty} \frac{\tau_{1}^{2}+\tau_{2}^{2}}{\left(\tau_{1}+\tau_{2}\right)^{2}}=\lim _{\tau_{2} \rightarrow \infty} \frac{\tau_{1}^{2}+\tau_{2}^{2}}{\left(\tau_{1}+\tau_{2}\right)^{2}}=1
$$

Le facteur de structure dynamique reste égal à l'unité, indépendamment du transfert de moment.

La transformation de Fourier par rapport au temps conduit à la fonction de diffusion dynamique

$$
\begin{aligned}
S(Q, \omega) & =\frac{1}{\left(\tau_{1}+\tau_{2}\right)^{2}}\left[\tau_{1}^{2}+\tau_{2}^{2}+2 \tau_{1} \tau_{2} \frac{\sin (Q . r)}{Q . r}\right] \delta(\omega) \\
& +\frac{2 \tau_{1} \tau_{2}}{\left(\tau_{1}+\tau_{2}\right)^{2}}\left[1-\frac{\sin (Q . r)}{Q \cdot r}\right] \frac{1}{\pi} \frac{\tau}{1+\omega^{2} \tau^{2}}
\end{aligned}
$$




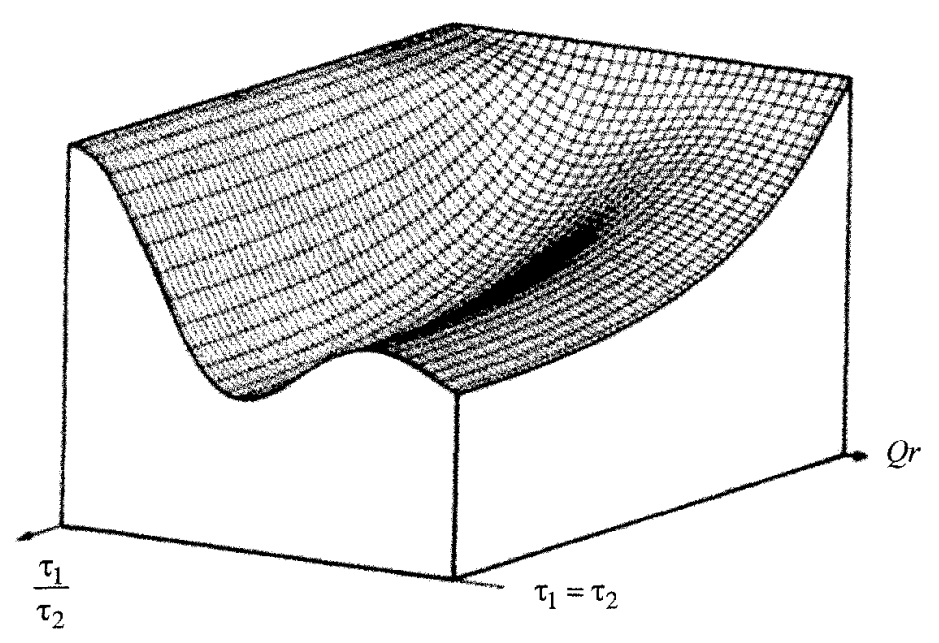

Figure 12: Evolution de l'EISF pour le modèle de sauts sur deux sites non équivalents

Conformément aux propriétés générales des fonctions de diffusion présentées précédemment, la fonction de diffusion est formée d'un pic élastique de largeur nulle, superposé à une fonction lorentzienne de largeur $1 / \tau$. La détermination des intensités respectives des intensités diffusées élastique et quasiélastique conduit à une détermination expérimentale de l'EISF.

\subsection{Exemple d'application: dynamique des protéines}

Le modèle de sauts sur deux sites non équivalents a été utilisé pour étudier la transition dynamique de la myoglobine hydratée par $\mathrm{D}_{2} \mathrm{O}$ [29]. De $4 \mathrm{~K}$ à $180 \mathrm{~K}$ l'intensité élastique évolue avec le transfert de moment selon la loi Gaussienne attendue pour un solide harmonique dont les vibrations peuvent se décrire par un facteur de Debye-Waller

$$
S(Q, \omega=0)=\exp \left(-Q^{2}<^{2}>\right)
$$

avec un déplacement carré moyen $\left\langle{ }^{2}>\right.$ proportionnel à la température. Mais au voisinage de $200 \mathrm{~K}$ la décroissance de l'intensité aux faibles valeurs de $Q$ s'accentue, indiquant l'apparition de nouveaux degrés de liberté. La géométrie de ces mouvements donne lieu à un écart au comportement gaussien qui augmente avec la température, selon la loi:

$$
S(Q, \omega=0)=\frac{\exp \left(-Q^{2}<^{2}>\right)}{\left(\tau_{1}+\tau_{2}\right)^{2}}\left[\tau_{1}^{2}+\tau_{2}^{2}+2 \tau_{1} \tau_{2} \frac{\sin (Q . r)}{Q . r}\right]
$$

La dérivée à $Q=0$ est la pente initiale de la courbe. Elle fait intervenir la géométrie du système (distance entre les sites) et les temps de résidence dans chaque site 


$$
-\left(\frac{d \ln \{S(Q, 0)\}}{d\left(Q^{2}\right)}\right)_{Q=0}=<^{2}>+\frac{\tau_{1} \tau_{2}}{\left(\tau_{1}+\tau_{2}\right)^{2}} \frac{r^{2}}{3}
$$

En affinant l'équation précédente (129) aux données expérimentales,il est possible d'obtenir $\left\langle{ }^{2}\right\rangle, r^{2}$ ainsi que le rapport $\tau_{1} / \tau_{2}$. Cette dernière valeur est reliée à l'énergie libre $\Delta \mathrm{G}$, par une relation simple

$$
\frac{\tau_{1}}{\tau_{2}}=\exp (-\Delta G / R T)
$$

Dans le cas de la myoglobine, Doster et coll. [29] en déduisent une asymétrie du puits $\Delta H=12 \mathrm{~kJ} \cdot \mathrm{mol}^{-1}$ et une entropie $\Delta S / R=3,0$. La distance de saut est $r=1.5 \AA$.

\subsection{Sauts sur trois sites équivalents}

L'expression du facteur de structure dynamique correspondant à des sauts sur trois positions équivalentes réparties sur un cercle de rayon $r$ est assez simple à obtenir selon la méthode de calcul exposée précédemment. Pour un échantillon polycristallin elle s'écrit:

$$
S^{3-\text { sites }}(Q, \omega)=\frac{1}{3}\left\{1+2 \frac{\sin (Q r \sqrt{3}}{Q r \sqrt{3}}\right\} \delta(\omega)+\frac{2}{3}\left\{1-\frac{\sin (Q r \sqrt{3}}{Q r \sqrt{3}}\right\} \frac{1}{\pi} \frac{\tau}{1+\omega^{2} \tau^{2}}
$$

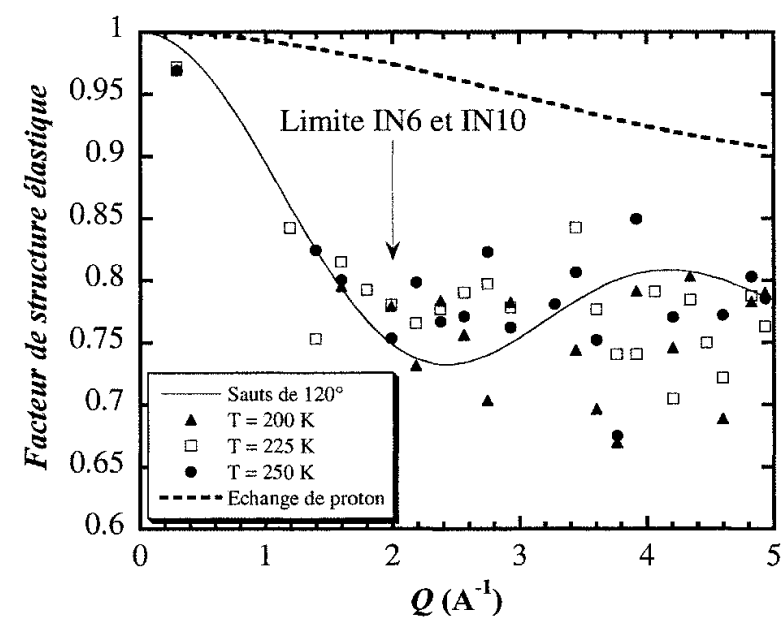

Figure 13: EISF déterminé à partir de la séparation des spectres obtenus avec le spectromètre IN13 entre leur partie purement élastique et leur partie quasiélastique, $(T=200 \mathrm{~K}, T=225 \mathrm{~K}, T=250 \mathrm{~K})$. La courbe continue est la variation théorique prédite par le modèle basé sur des sauts de $120^{\circ}$ des groupes méthyles. La courbe en tirets correspond à un autre modèle qui suppose un échange de proton le long de la liaison hydrogène.

La rotation des deux groupes méthyles de l'acide camphre sulfonique monohydraté, $\mathrm{C}_{10} \mathrm{H}_{15} \mathrm{O}_{4} \mathrm{~S}^{-}-\mathrm{H}_{3} \mathrm{O}^{+}$ (en abrégé: CSA), a été analysée par diffusion quasiélastique de 15 à $340 \mathrm{~K}$, par spectrométries de rétrodiffusion et temps de vol [11]. Le facteur de structure extrait expérimentalement des données 
recueillies avec le spectromètre IN13 est représenté sur la figure 13. En raison du faible flux de neutrons résultant de la configuration expérimentale de cette époque, les incertitudes sur les points expérimentaux sont particulièrement grandes. De plus, les hydrogènes en mouvement sont dans le rapport 6:18 par rapport au nombre total d'hydrogènes observables.

En tenant compte des 12 hydrogènes fixes qui diffusent de manière purement élastique, la loi de diffusion s'écrit

$$
\begin{aligned}
S(Q, \omega) & =\frac{12}{18} \delta(\omega)+\frac{6}{18} S^{3-\text { sites }}(Q, \omega) \\
& =\frac{1}{9}\left\{7+2 \frac{\sin (Q r \sqrt{3}}{Q r \sqrt{3}}\right\} \delta(\omega)+\frac{2}{9}\left\{1-\frac{\sin (Q r \sqrt{3}}{Q r \sqrt{3}}\right\} \frac{1}{\pi} \frac{\tau}{1+\omega^{2} \tau^{2}}
\end{aligned}
$$
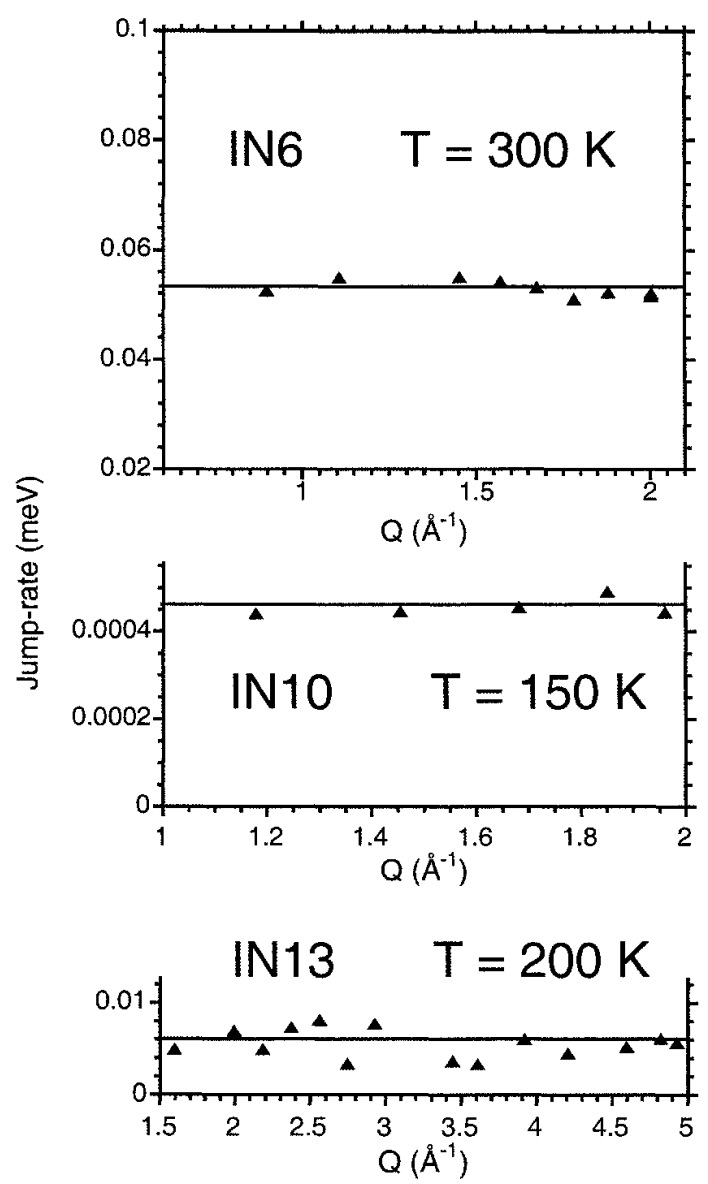

Figure 14: Largeur à mi-hauteur des spectres obtenus à partir des différents spectromètres. 
Elle est représentée en trait continu sur la figure 13 pour $r=1.07 \AA$. L'EISF reste toujours très élevé avec un minimum (0.73) pour $Q=2.5 \AA^{-1}$ lorsque $Q r \sqrt{3}=3 \pi / 2$. C'est pourquoi son extraction est délicate, surtout pour de faibles élargissements. La gamme en $Q$ accessible aux spectromètres IN10 et IN6 $(Q<$ $\left.2 \AA^{-1}\right)$ ne permet pas d'observer ce minimum. C'est pourquoi en dépit de son faible flux le spectromètre IN13 s'avère fort utile.

L'évolution de la variation de l'élargissement quasiélastique des spectres en énergie en fonction du transfert de moment est une autre information facile à extraire des spectres. Elle peut permettre de distinguer entre plusieurs modèles. Dans le cas général la partie quasiélastique de l'intensité diffusée s'exprime sous la forme d'un développement (parfois infini) en fonctions lorentziennes. Leurs largeurs dépendent explicitement d'un petit nombre de temps caractéristiques associés aux détails des mouvements moléculaires. Leurs contributions relatives changent avec $Q$ suivant les variations des facteurs de structure qui leur sont associés. Ainsi l'élargissement global des spectres varie avec le transfert de moment. Il existe cependant certains cas où cet élargissement reste strictement constant, en particulier pour les modèles de sauts sur deux ou trois sites. Dans l'exemple du CSA, les deux modèles sont envisageables: rotation de $120^{\circ}$ des groupes méthyles ou passage du proton de la liaison hydrogène entre deux sites. La figure 14 montre clairement que la largeur à mi-hauteur de la partie quasiélastique des spectres reste constante avec $Q$, à toutes les températures c'est-à-dire pour toutes les échelles de temps. Ceci est particulièrement remarquable avec le spectromètre IN13 qui offre la plus grande gamme de transfert de moment $\left(1.5-5 \AA^{-1}\right)$. L'évolution de l'EISF permet de lever l'ambiguité.

\subsection{Le balayage élastique (elastic scan): la méthode "fenêtre fixe" (fixed window)}

Avec les spectromètres à rétrodiffusion, on utilise couramment une méthode appelée "fenêtre fixe". Avec cette technique on envoie sur l'échantillon des neutrons qui ont exactement l'énergie sélectionnée par les analyseurs. De la sorte, seuls les neutrons diffusés sans changement d'énergie (dans la limite de la résolution expérimentale) sont enregistrés. Dans le même temps, on fait varier un paramètre physique extérieur, en général la température, parfois la pression.

L'apparition d'un mouvement dont la dynamique se situe dans la fenêtre expérimentale se manifeste par un élargissement quasiélastique et conduit à une baisse de l'intensité élastique détectée [30,31]. Cette technique permet une inspection rapide de la dynamique de l'échantillon dans un vaste domaine de température. Bien entendu, ces observations doivent être complétées par des mesures quasiélastiques afin d'obtenir une description complète des mouvements du système.

La figure 15 montre sur une échelle logarithmique l'évolution de l'intensité élastique avec la température, telle qu'elle a été mesurée pour le CSA avec le spectromètre IN10. On distingue clairement trois régions:

i) A basse température $(T<100 \mathrm{~K})$ tous les mouvements moléculaires diffusifs sont trop lents pour donner lieu à un quelconque élargissement des spectres quasiélastiques. La contribution inélastique est si faible qu'on peut la négliger. L'intensité enregistrée est purement élastique. Elle évolue avec la température selon la loi

$$
S(Q, \omega=0)=C^{s t e} \exp \left(-Q^{2}<u^{2}>\right)
$$

A chaque température il est possible d'extraire à partir de la dépendance de l'intensité en fonction de $Q$ un déplacement carré moyen $\left\langle u^{2}(T)>\right.$ qui décrit les mouvements purement harmoniques. La figure 15 montre qu'il varie linéairement avec la température.

ii) A plus haute température $(100 \mathrm{~K}<T<170 \mathrm{~K})$, l'intensité élastique décroît plus rapidement en raison de l'apparition d'une composante quasiélastique plus large que la résolution du spectromètre. En utilisant l'expression (134) ci-dessus, on peut extraire un déplacement carré moyen "effectif", qui tient compte à la fois des mouvements vibrationnels et diffusifs et qui est une grossière estimation de leur amplitude. Cette approche est couramment utilisée dans l'étude de la dynamique des macromolécules biologiques [32]. 
iii) Pour $T>170 \mathrm{~K}$, lélargissement quasiélastique devient trop large pour la gamme en énergie du spectromètre $(-10 \mu \mathrm{eV},+10 \mu \mathrm{eV})$. La majeure partie de l'intensité diffusée est à l'extérieur de cette fenêtre et l'intensité enregistrée autour du pic élastique devient de moins en moins sensible à l'augmentation de l'élargissement avec la température. Elle évolue de nouveau selon le terme de Debye-Waller de la relation (134). Sur la figure 15 sont représentées pour chaque angle deux droites obtenues par affinement aux données expérimentales. Elles ne se croisent pas exactement à $T=0$. Ceci provient du choix plus ou moins arbitraire des limites des domaines en température. On observe également une différence notable entre les deux pentes, ce qui s'explique par l'évolution exacte de la composante quasiélastique avec la température et par la nature plus complexe du terme $\left\langle u^{2}(T)\right\rangle$ à haute température. Cette analyse est décite en détails dans les références $[30,31]$

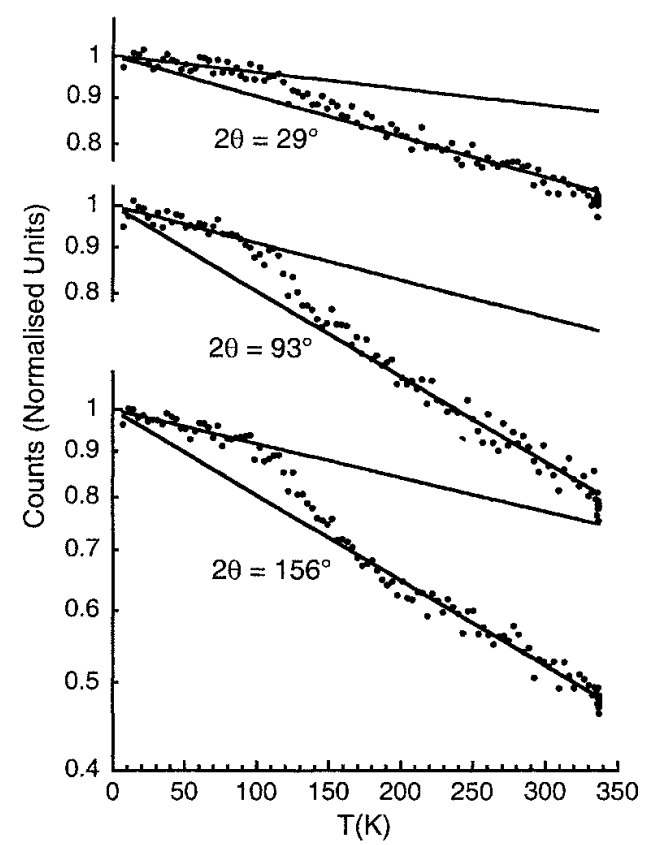

Figure 15: Evolution avec la température de l'intensité purement élastique pour différentes valeurs du transfert de moment obtenue par un balayage élastique avec le spectromètre IN10. Les lignes droites représentent les variations théoriques prédites par le terme de Debye-Walleur seul. Elles ont été affinées aux données expérimentales respectivement à $T<100 \mathrm{~K}$ et $T\rangle$ $170 \mathrm{~K}$. Les intensités des différents détecteurs sont toutes normalisées à $T=0 \mathrm{~K}$.

A partir des données de ce type d'expériences, et en supposant que l'élargissement quasiélastique est suffisamment important au dessus de $T=170 \mathrm{~K}$ pour donner lieu à un bruit de fond pratiquement constant, il est possible d'extraire une estimation grossière de l'EISF. Nous renvoyons à [11] pour plus d'explication

\subsection{Application de la méthode "fenêtre fixe" aux composés biologiques}

Les interactions avec le solvant participent à la stabilisation de la structure d'une protéine via l'hydratation, les forces de van der Waals, les liaisons hydrogène, les liaisons entre ions et les effets hydrophobiques. Puisque ces mêmes forces contrôlent les fluctuations thermiques, on imagine intuitivement une relation entre ces interactions, la stabilité de la protéine et la dynamique: une protéine plus molle, plus flexible devrait être moins stable. Cependant une plus faible flexibilité n'est pas toujours synonyme de stabilité. Quand les termes entropiques dominent, une protéine flexible peut être plus stable. 
Une étude récente porte sur le comportement dynamique en fonction de la température de la protéine halophile malate déshydrogénase Haloarcula marismortui ( $\mathrm{Hm} \mathrm{MalDH})$ dans différentes conditions de solvant [33]. La disparition de la structure secondaire, choisie comme critère de stabilité thermique, a été déterminée par dichroïsme circulaire. A partir de la dérivée de la courbe expérimentale représentant $\left\langle u^{2}\right\rangle$ en fonction de la température, il est possible de calculer une constante de force effective moyenne, caractérisant la résilience moyenne de la structure de la protéine [32]:

$$
<k^{\prime}>=\frac{0,00276}{\frac{d<u^{2}>}{d T}}
$$

La constante numérique permet d'exprimer $\left\langle k^{\prime}\right\rangle$ en newton/mètre $(\mathrm{N} / \mathrm{m})$ si $\left.<u^{2}\right\rangle$ est exprimé en $\AA^{2}$ et $T$ en Kelvins. Dans le cas de $H m \mathrm{MalDH}$ la résilience (inverse de la pente sur la figure 16) augmente progressivement avec la stabilité, en passant d'une solution molaire de $\mathrm{NaCl}$ dans $\mathrm{H}_{2} \mathrm{O}$ à une solution molaire de $\mathrm{KCl}$ dans $\mathrm{D}_{2} \mathrm{O}$ puis à une solution molaire de $\mathrm{NaCl}$ dans $\mathrm{D}_{2} \mathrm{O}$. Ce comportement montre le rôle dominant des termes liés à l'enthalpie provenant des interactions de liaisons.

\section{Température $(\mathrm{C})$}

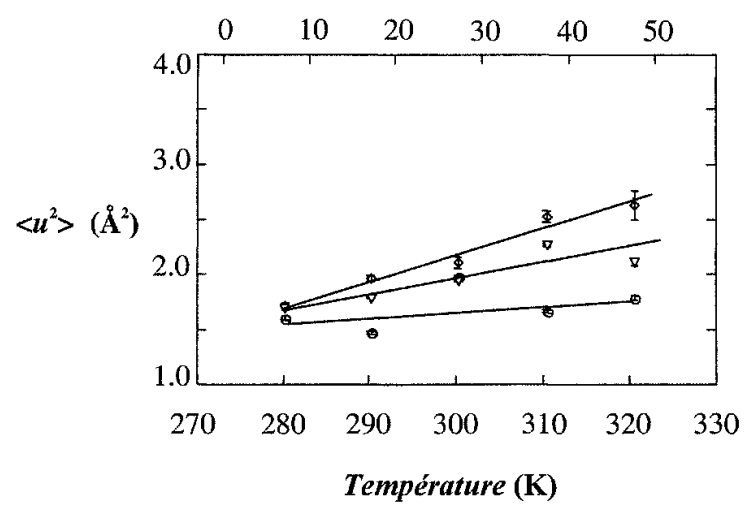

Figure 16: Evolution avec la température du déplacement carré moyen pour HmMALDH dans différents solvants. La résilience de la protéine (inverse de la pente de la droite) est de plus en plus faible dans les mélanges molaires $\mathrm{NaCl} / \mathrm{D}_{2} \mathrm{O}$, $\mathrm{KCl} / \mathrm{D}_{2} \mathrm{O}, \mathrm{NaCl} / \mathrm{H}_{2} \mathrm{O}$ dans lesquels elle est de moins en moins stable.

On observe un comportement opposé avec l'albumine de sérum bovin. Sa résilience est plus élevée dans $\mathrm{H}_{2} \mathrm{O}$ où elle est moins stable que dans $\mathrm{D}_{2} \mathrm{O}$, ce qui montre que sa plus grande stabilité thermique est due à des fluctuations entropiques. Ces résultats confirment la complexité des relations dynamique-stabilité dans les protéines.

\section{LA SITUATION ACTUELLE DE LA DIFFUSION INELASTIQUE}

Les premières applications de la diffusion quasiélastique des neutrons portaient sur des systèmes relativement simples dans lesquels il était possible de décrire avec précision à la fois les dimensions moléculaires et la géométrie des mouvements. Ces expériences ont suscité nombre de modèles dynamiques pour lesquels les lois de diffusion correspondantes pouvaient être déduites sans difficultés majeures. La plupart de ces modèles concernaient des rotations de molécules ou de groupes chimiques. L'utilisation d'échantillons bien cristallisés a permis de développer des approches mathématiques basées sur la symétrie. Toutes ces études ont mis en évidence l'importance de l'EISF qui est rapidement apparu comme l'information primordiale à tirer des données expérimentales. De nos jours on s'intéresse à des 
échantillons beaucoup plus compliqués. Cette complexité peut être le résultat de la molécule elle-même (les polymères ou les macromolécules biologiques offre un large éventail de mouvements possibles qui peuvent se produire sur des échelles de temps différentes) mais aussi de l'état physique dans lequel se trouve l'échantillon: verres, phases cristalline amorphes ou semi-ordonnées, composé d'intercalation, dilution dans un liquide... De nombreux effets rendent souvent le milieu inhomogène. En raison de différences dans leur environnement local, les groupements chimiques identiques ne présenteront pas la même dynamique. Les valeurs de l'EISF que l'on peut extraire des données expérimentales dépendent de la résolution expérimentale et leur signification physique n'est pas claire.

Des modèles théoriques ont été développés pour tenir compte de telles situations, comme la théorie du couplage de modes (mode-coupling). Ils se présentent souvent sous une forme abstraite et peuvent difficilement être reliés aux données. Ils sont en général basés sur une description phénoménologique du comportement du système. Ainsi il est possible de vérifier leurs prédictions quant au comportement de la fonction de diffusion, mais une description de la dynamique en termes de distances de saut ou de temps caractéristiques de molécules individuelles est loin d'être évidente. Les simulations de dynamique moléculaire semblent prometteuses à court terme. Mais elles demandent des temps de calcul particulièrement longs et pour cela elles sont souvent utilisées pour des petites molécules. Avant tout il est indispensable de disposer d'un potentiel d'interaction fiable. Dans certains cas (présence de charges délocalisées), cette prescription peut constituer une sérieuse difficulté.

On a pu constater ces dernières années un effort important pour améliorer l'analyse des données. En particulier pour accéder à la fonction de diffusion intermédiaire qui est la véritable grandeur pertinente. Celle dernière est directement accessible par la spectroscopie par écho de spin. En principe elle devrait pouvoir aussi d'obtenir par transformée de Fourier inverse des spectre en énergie obtenus par temps de vol ou rétrodiffusion. Cette méthode présente en fait de nombreuses difficultés liées à la résolution expérimentale et à la gamme d'énergie restreinte explorée par chaque instrument.

D'un point de vue expérimental, l'analyse d'un échantillon compliqué doit être conduite en multipliant les conditions expérimentales: température, pression, concentration, deutération sélective...Mais aussi il s'avère important de collecter le maximum d'informations sur le système, à partir d'autres techniques (RMN, simulations numériques...).

\section{Références}

[1] Egelstaff P.A., Thermal Neutron Scattering (Academic, New-York, 1971).

[2] Lovesey S.W., Theory of Neutron Scattering from Condensed Matter (Clarendon, Oxford, 1984).

[3] Squires G.L., Introduction to the Theory of Thermal Neutron Scattering (Cambridge University Press, Cambridge, 1978)

[4] Willis B.T.M., Chemical Applications of Thermal Neutron Scattering (Oxford University Press, 1973)

[5] Kostorz G., Neutron Scattering; Treatise on Materials Science and Technology Vol.15 (Academic Press, New-York, 1979)

[6] Bée M., Quasielastic Neutron Scattering; Principles and Applications in Solid State Chemistry, Biology and Materials Science (Adam Hilger, Bristol, 1988)

[7] Van Hove, Phys. Rev. 1954, 95 (1), 249-262.

[8] Glauber, R. Phys. Rev. 1955, 98 (6), 1692-1698.

[9] Zemach, A.J.; Glauber, R. Phys. Rev. 101,1 (1956) 118-136.

[10] Gaigeot, M.-P., Leulliot, N., Ghomi, M., Jobic, H. Coulombeau, C. Bouloussa, O., Chemical Physics 261 (2000) 217

[11] Bée, M., Djurado, D. Combet, J, Gonzalez, M.A. Chemical Physics 277 (2002) 211

[12] Frick, B.; Farago, B. Neutron Spectroscopy by Time-of-Flight, backscattering and Spin-Echo Techniques. In Scattering; Scattering and Inverse Scattering in Pure and Applied Science, Pike, R., Sabatier, P., Eds.; Academic Press: San Diego San Francisco New York Boston London Sydney Tokyo, 2002; 1209-1241.

[13] http://www.ill.fr (accessed May 2002)

[14] http://www-llb.cea.fr (accessed May 2002) 
[15] http://www.hmi.de (accessed May 2002)

[16] http://rddjazz.nist.gov (accessed May 2002)

[17] http://www.psi.ch (accessed May 2002)

[18] http://www.isis.rl.ac.uk (accessed May 2002)

[19] Mezei, F., Z. Physik 255 (1972) 146.

[20] Mezei, F., (Ed.) Lecture Notes in Physics: Neutron Spin Echo, Vol. 128, Springer Verlag BerlinHeidelberg-New York, 1980.

[21] Golub, R. and Gähler, R., Physics Letters A, 123, (1987) 43.

[22] Egelstaff P. A., An Introduction to the Liquid State (Academic, London, 1967)

[23] Hempelmann R. Quasielastic Neutron Scattering and Solid State Diffusion (Clarendon Press, Oxford, 2000)

[24] Einstein A., Ann. Phys. 17 (1905) 349

[25] Chudley C. T., Elliott R. J., Proc. Phys. Soc. London 77 (1961) 353

[26] Hall P. L., Ross D. K., Mol. Phys. 42 (1981) 673

[27] Singwi K.S., Sjölander A. Phys. Rev. 119 (1960) 863

[28] Jobic, H. J. Physique IV 10 (2000) 77

[29] Doster, W. Cusack, S. and Petry, W. Nature 1989, 337754.

[30] Frick, B. Fetters, L.J. Macromolcules 27 (1994) 974.

[31] Frick, B., Magerl, A., Blanc, Y., Rebesco, R. Physica B 234-236 (1990) 1177

[32] Zaccai, G. Science, 288 (2000) 1604-1607

[33] Tehei M., Madern, D., Pfister, C. Zaccaï, J. PNAS 98, 25 (2001) 14356-14361 